\title{
Global stability results for systems under sampled-data control
}

\author{
Iasson Karafyllis ${ }^{1}$ and Costas Kravaris ${ }^{2, *, \dagger}$ \\ ${ }^{1}$ Department of Environmental Engineering, Technical University of Crete, 73100 Chania, Greece \\ ${ }^{2}$ Department of Chemical Engineering, University of Patras, 1 Karatheodory Str., 26500 Patras, Greece
}

\begin{abstract}
SUMMARY
In this work sufficient conditions for uniform input-to-output stability and uniform input-to-state stability are presented for finite-dimensional systems under feedback control with zero-order hold. The conditions are expressed by means of single and vector Lyapunov functions. Copyright (C) 2008 John Wiley \& Sons, Ltd.
\end{abstract}

Received 25 October 2006; Revised 16 June 2008; Accepted 16 June 2008

KEY WORDS: Lyapunov functions; sampled-data control; input-to-output stability

\section{INTRODUCTION}

When a feedback controller is designed for a continuous-time nonlinear system but it is actually implemented in discrete time under sampled measurements and zero-order hold, the resulting closed-loop system does not necessarily possess the same properties as the closed-loop system under continuous-time implementation. The deduction of properties under discrete-time feedback from properties under continuous-time feedback is quite difficult and involved. Questions such as

'Can a state feedback robustly globally asymptotically stabilize the origin under zero-order hold discrete-time implementation, if it globally asymptotically stabilizes the origin under continuous-time implementation?'

do not have an affirmative answer in general, even if the sampling period is assumed to be arbitrarily small. In addition, in case the answer to the above question is affirmative, the next important question is

'What is the maximum allowable sampling period for guaranteed global asymptotic stability?'

In general, providing answers to the above questions is a highly non-trivial task. There is a large body of literature concerning the foregoing very important and very challenging issues. In

\footnotetext{
*Correspondence to: Costas Kravaris, Department of Chemical Engineering, University of Patras, 1 Karatheodory Str., 26500 Patras, Greece.

†E-mail: kravaris@chemeng.upatras.gr
} 
particular, the following lines of attack have been pursued to derive stability results (see also the detailed discussion in review paper [1]):

- making use of numerical approximations of the solution of the open-loop system (e.g. in the work of D. Nesic, A. Teel and others, see [2-13]). The results obtained in this way lead to a systematic procedure for the construction of practical, semi-global feedback stabilizers and provide a list of possible reasons that explain the occasional failure of sampled-data control mechanisms. Recent research takes into account performance and robustness issues as well (see $[2,12,14,15])$;

- exploiting special characteristics of the system such as homogeneity (see [16]), global Lipschitz conditions (see [17]) or linear structure with uncertainties (see [18] as well as the textbook [19]);

- making use of linear matrix inequalities in the context of hybrid systems (see [20-23]);

- considering the closed-loop system as a discrete-time system (see, for instance, [24, 25] and the paper [26], which establishes a unified representation for sampled-data systems and discretetime systems with analytic dynamics). Recent work has established results that characterize the inter-sample behavior of the solutions based on the behavior of the solution of the discretetime system (see [6]).

It is important to note that the above important research results do not provide conditions for global asymptotic stability or input-to-output stability for general nonlinear sampled-data systems (usually only semi-global practical stability properties are established or global stability for limited classes of systems). One exception is the recent paper [27], which utilizes recent results for hybrid systems.

A very interesting point of view that was recently explored in the linear systems theory (see $[28,29]$ ) involves considering the closed-loop system under zero-order hold as a time-delay system. This is a natural and intuitively meaningful point of view, since sampled-data control introduces piecewise constant retarded arguments. It is exactly this point of view that will be explored in the present work for nonlinear systems for the first time.

Moreover, the present work will utilize the method of Razumikhin functions (see [30-32]) for stability analysis of time-delay systems, together with recent developments in the theory of vector Lyapunov functions (see [33]). The main contribution will be the development of Lyapunovlike sufficient conditions for uniform input-to-output stability (UIOS) and uniform input-to-state stability (UISS). The notions of UIOS and UISS were formulated in [34-38] for finite-dimensional systems described by ordinary differential equations. More recently, sufficient conditions for semiglobal practical UISS were studied in $[4,5]$ for sampled-data systems. In the present work, the sufficient conditions will be expressed in terms of a scalar Lyapunov function and a vector Lyapunov function. The main features of the results will be that they

- can be applied to uncertain nonlinear systems with no special characteristics (such as homogeneity or global Lipschitz conditions);

- can lead to global stability results for an arbitrary output map (including the possibility of using the entire state vector as output and thus recovering the familiar stability notions for the state);

- do not require knowledge of the solution map for the open-loop system;

- allow the direct determination of the maximum allowable sampling period;

- guarantee robustness to perturbations of the sampling schedule. 
The present work does not aim directly at the construction/design of sampled-data feedback stabilizers. The main goal is to develop Lyapunov-like sufficient conditions for robust global asymptotic stability or input-to-output stability of (general nonlinear) sampled-data systems. These Lyapunov-like sufficient conditions in the present work can be potentially useful for the construction and/or the Lyapunov redesign of sampled-data stabilizers for nonlinear control systems (see [12]), although this application will not be addressed in the present work. We have just become aware of related results in [27], where the approach and methodology is very different from the approach adopted in the present work.

The structure of the present paper is as follows: In Section 2, we state the problem to be studied and in Section 3 we state the main assumptions to be made. Section 4 is devoted to the statement and the proof of the main results. In Section 5, illustrating examples are presented, which demonstrate the flexibility that a vector Lyapunov function can provide. Moreover, we show how the results of Section 4 can be used in conjunction with the backstepping method for triangular nonlinear systems to check whether the feedback, constructed by the backstepping method presented in [39], can robustly globally asymptotically stabilize the equilibrium point when applied with zero-order hold and positive sampling rate. Finally, Section 6 contains some concluding remarks of the present work.

\section{PROBLEM STATEMENT}

Consider the finite-dimensional continuous-time system:

$$
\begin{aligned}
\dot{x}(t) & =F(x(t), u(t), d(t)) \\
Y(t) & =H(x(t)) \\
x(t) & \in \Re^{n}, \quad u(t) \in \mathfrak{R}^{m}, \quad d(t) \in D \subseteq \Re^{l}, \quad Y(t) \in \mathfrak{R}^{k}
\end{aligned}
$$

where the vector field $F: \Re^{n} \times U \times D \rightarrow \Re^{n}$ is continuous, $u(t)$ represents the control input and $d(t)$ unknown disturbances or model uncertainty. Suppose now that a continuous-time control law $u(t)=k(x(t))$ is applied to system (1). Then the resulting closed-loop system can be analyzed and the feedback function can be selected for desirable properties, e.g. robust global asymptotic stability or input-to-state stability.

When the foregoing control law is implemented in discrete time under constant sampling period $r$ and zero-order hold

$$
\begin{aligned}
& u(t)=k\left(x\left(\tau_{i}\right)\right), \quad t \in\left[\tau_{i}, \tau_{i+1}\right) \\
& \tau_{i+1}=\tau_{i}+r, \quad i=0,1,2, \ldots
\end{aligned}
$$

the resulting closed-loop system is the following switched system:

$$
\begin{aligned}
\dot{x}(t) & =F\left(x(t), k\left(x\left(\tau_{i}\right)\right), d(t)\right), \quad t \in\left[\tau_{i}, \tau_{i+1}\right) \\
\tau_{i+1} & =\tau_{i}+r, \quad i=0,1,2, \ldots \\
Y(t) & =H(x(t))
\end{aligned}
$$

and the question is under what conditions will the origin be robustly globally asymptotically stable for system (3). 
In this work, we will actually consider a broader class of switched systems, described in the following manner: given a pair of sets $D \subseteq \Re^{l}$, a positive function $h: \Re^{n} \times U \rightarrow(0, r]$, which is bounded by a certain constant $r>0$ and a pair of vector fields $f: \Re^{n} \times \Re^{n} \times D \times U \times U \rightarrow \Re^{n}$, $H: \mathfrak{R}^{n} \rightarrow \mathfrak{R}^{k}$, we consider the switched system that produces for each $\left(t_{0}, x_{0}\right) \in \mathfrak{R}^{+} \times \mathfrak{R}^{n}$ and for each triplet of measurable and locally bounded inputs $d: \mathfrak{R}^{+} \rightarrow D, \tilde{d}: \mathfrak{R}^{+} \rightarrow \mathfrak{R}^{+}, v: \mathfrak{R}^{+} \rightarrow U$ the piecewise absolutely continuous function $t \rightarrow x(t) \in \mathfrak{R}^{n}$, via the following algorithm:

Step $i$ :

(1) Given $\tau_{i}$ and $x\left(\tau_{i}\right)$, calculate $\tau_{i+1}$ using the equation $\tau_{i+1}=\tau_{i}+\exp \left(-\tilde{d}\left(\tau_{i}\right)\right) h\left(x\left(\tau_{i}\right), v\left(\tau_{i}\right)\right)$.

(2) Compute the state trajectory $x(t), t \in\left[\tau_{i}, \tau_{i+1}\right)$ as the solution of the differential equation $\dot{x}(t)=f\left(x(t), x\left(\tau_{i}\right), d(t), v(t), v\left(\tau_{i}\right)\right)$.

(3) Calculate $x\left(\tau_{i+1}\right)$ using the equation $x\left(\tau_{i+1}\right)=\lim _{t \rightarrow \tau_{i+1}^{-}} x(t)$.

For $i=0$ we take $\tau_{0}=t_{0}$ and $x\left(\tau_{0}\right)=x_{0}$ (initial condition). Schematically, the algorithm is expressed by

$$
\begin{aligned}
\dot{x}(t) & =f\left(x(t), x\left(\tau_{i}\right), d(t), v(t), v\left(\tau_{i}\right)\right), \quad t \in\left[\tau_{i}, \tau_{i+1}\right) \\
\tau_{0} & =t_{0}, \tau_{i+1}=\tau_{i}+\exp \left(-\tilde{d}\left(\tau_{i}\right)\right) h\left(x\left(\tau_{i}\right), v\left(\tau_{i}\right)\right), \quad i=0,1, \ldots \\
Y(t) & =H(x(t))
\end{aligned}
$$

with initial condition $x\left(t_{0}\right)=x_{0}$. Switched systems of the form (4) will be called 'sampled-data' systems. The objective of the present work is to derive Lyapunov-like sufficient conditions for UIOS for systems of the form (4).

Note that systems of the form (4) arise as closed-loop finite-dimensional systems under feedback control with zero-order hold. Specifically, consider the finite-dimensional continuous-time control system (1). Then, the application of the (not necessarily continuous) feedback law

$$
\begin{aligned}
u(t)= & k\left(x\left(\tau_{i}\right)+e\left(\tau_{i}\right)\right)+w(t) \quad \text { on the interval }\left[\tau_{i}, \tau_{i}+\exp \left(-\tilde{d}\left(\tau_{i}\right)\right) \tilde{h}\left(x\left(\tau_{i}\right)+e\left(\tau_{i}\right)\right)\right) \\
& i=0,1,2, \ldots
\end{aligned}
$$

where $w(t) \in U \subseteq \Re^{m}$ represents the control actuator error, $e(t) \in \mathfrak{R}^{n}$ represents the measurement error, $\tilde{h}: \Re^{n} \rightarrow(0, r]$ is a positive function bounded by certain constant $r>0$ and $\tilde{d}(t) \geqslant 0$ is the input that quantifies the perturbation of the sampling schedule, gives rise to the dynamical system, that produces for each $\left(t_{0}, x_{0}\right) \in \mathfrak{R}^{+} \times \mathfrak{R}^{n}$ and for each measurable and locally bounded inputs $d: \mathfrak{R}^{+} \rightarrow$ $D, w: \Re^{+} \rightarrow U, e: \Re^{+} \rightarrow \Re^{n}$ and $\tilde{d}: \Re^{+} \rightarrow \Re^{+}$the absolutely continuous function $\left[t_{0},+\infty\right) \ni t \rightarrow$ $x(t) \in \Re^{n}$ that satisfies a.e. the differential equation

$$
\begin{aligned}
\dot{x}(t) & =F\left(x(t), k\left(x\left(\tau_{i}\right)+e\left(\tau_{i}\right)\right)+w(t), d(t)\right), \quad t \in\left[\tau_{i}, \tau_{i+1}\right) \\
\tau_{0} & =t_{0}, \tau_{i+1}=\tau_{i}+\exp \left(-\tilde{d}\left(\tau_{i}\right)\right) \tilde{h}\left(x\left(\tau_{i}\right)+e\left(\tau_{i}\right)\right), \quad i=0,1, \ldots \\
Y(t) & =H(x(t))
\end{aligned}
$$

with initial condition $x\left(t_{0}\right)=x_{0}$. Clearly, system (6) is a system of the form (4) with $v=$ $(w, e), h(x, v):=\tilde{h}(x+e)$ and $f\left(x, x_{0}, d, v, v_{0}\right)=F\left(x, k\left(x_{0}+e_{0}\right)+w, d\right)$.

Some comments are in order relative to the discrete-time feedback law (5). In addition to accounting for actuator and measurement errors, (5) is a generalization of (2) in the following manners:

(i) The sampling period is allowed to be state dependent and input dependent. The situation of a non-constant $\tilde{h}: \Re^{n} \rightarrow(0, r]$ has recently emerged in connection with the study of asymptotic controllability of nonlinear systems (see the classical work in $[40,41]$ and the links with 
sampled-data stabilizability given in [42]). The reader should also note that state-dependent sampling periods arise when studying (a) event-triggered real-time scheduling of stabilizing control tasks (see the discussion in [43]), which are important for physically distributed actuator/sensor networks and (b) practical implementation of numerical schemes for the numerical solution of dynamical systems (see [44]). Of course, the situation of $\tilde{h}(x) \equiv r>0$ (constant) is very important, in which case we say that the sampled-data feedback law is applied with positive sampling rate (see [42]).

(ii) The sampling period is allowed to be time varying and uncertain. The factor $\exp \left(-\tilde{d}\left(\tau_{i}\right)\right) \leqslant 1$, with $\tilde{d}(t) \geqslant 0$ some non-negative function of time, is an uncertainty factor in the end-point of the sampling interval. Proving stability for any non-negative input $\tilde{d}: \mathfrak{R}^{+} \rightarrow \mathfrak{R}^{+}$will guarantee stability for all sampling schedules with $\tau_{i+1}-\tau_{i} \leqslant h\left(x\left(\tau_{i}\right), v\left(\tau_{i}\right)\right)$ (robustness to perturbations of the sampling schedule). To understand the importance of robustness to perturbations of the sampling schedule, consider the following situation. Suppose that the sampling period is $1 s$ (a hardware specification). If we manage to design a sampled-data feedback law with $h(x, v) \equiv r \geqslant 2 s$, then the implementation of the feedback control with sampling period $1 s$ will guarantee stability properties for the closed-loop system even if we 'miss measurements' or if we have 'delayed measurements' (for example, due to improper operation of the sensor). In such a case robustness to perturbations of the sampling schedule becomes critical. The introduction of the factor $\exp \left(-\tilde{d}\left(\tau_{i}\right)\right) \leqslant 1$ is a mathematical way of introducing perturbations to the sampling schedule; however, it is not unique. Other ways of introducing perturbations of the sampling schedule can be considered.

Notations: Throughout this paper we adopt the following notations:

- Let $I \subseteq \Re$ be an interval. By $C^{0}(I ; \Omega)$ we denote the class of continuous functions on $I$, which take values in $\Omega$. By $C^{1}(I ; \Omega)$ we denote the class of functions on $I$ with continuous derivative, which take values in $\Omega$.

- For a vector $x \in \Re^{n}$ we denote by $|x|$ its usual Euclidean norm and by $x^{\prime}$ its transpose.

- We denote by $K^{+}$the class of positive $C^{0}$ functions defined on $\mathfrak{R}^{+}$. We say that a nondecreasing continuous function $\gamma: \mathfrak{R}^{+} \rightarrow \mathfrak{R}^{+}$is of class $\mathscr{N}$ if $\gamma(0)=0$. We say that a function $\rho: \mathfrak{R}^{+} \rightarrow \mathfrak{R}^{+}$is positive definite if $\rho(0)=0$ and $\rho(s)>0$ for all $s>0$. For the definitions of the classes $K, K_{\infty}$ and $K L$, see [45].

- Let $U \subseteq \Re^{m}$ be a non-empty set with $0 \in U$. By $B_{U}[0, r]:=\{u \in U ;|u| \leqslant r\}$ we denote the closed sphere in $U \subseteq \Re^{m}$ with radius $r \geqslant 0$, centered at $0 \in U$.

- Let $D \subseteq \Re^{l}$ be a non-empty set. By $M(D)$ we denote the class of all Lebesgue measurable and locally bounded mappings $d: \Re^{+} \rightarrow D$. Note that members of $M(D)$ are functions $d: \Re^{+} \rightarrow D$, which are defined pointwise and are not equivalent classes of functions.

\section{MAIN ASSUMPTIONS AND PRELIMINARIES FOR SAMPLED-DATA SYSTEMS}

In the present work we study systems of the form (4) under the following hypotheses:

(H1) $f\left(x, x_{0}, d, v, v_{0}\right)$ is continuous with respect to $(x, d, v) \in \Re^{n} \times D \times U$ and such that for every bounded $S \subset \Re^{n} \times \Re^{n} \times U \times U$ there exists constant $L \geqslant 0$ such that

$$
\begin{aligned}
& (x-y)^{\prime}\left(f\left(x, x_{0}, d, v, v_{0}\right)-f\left(y, x_{0}, d, v, v_{0}\right)\right) \leqslant L|x-y|^{2} \quad \forall\left(x, x_{0}, v, v_{0}, d\right) \in S \times D \\
& \forall\left(y, x_{0}, v, v_{0}, d\right) \in S \times D
\end{aligned}
$$


Hypothesis (H1) is a standard continuity hypothesis and condition (7) is characterized as a 'onesided Lipschitz condition on compact sets' in the literature (see [46]). Note that we do not assume continuity of the vector field $f\left(x, x_{0}, d, v, v_{0}\right)$ with respect to $x_{0} \in \Re^{n}$ and $v_{0} \in U$.

(H2) There exists a function $a \in K_{\infty}$ such that

$$
\left|f\left(x, x_{0}, d, v, v_{0}\right)\right| \leqslant a\left(|x|+\left|x_{0}\right|+|v|+\left|v_{0}\right|\right) \quad \forall\left(v, v_{0}, d, x, x_{0}\right) \in U \times U \times D \times \Re^{n} \times \Re^{n}
$$

(H3) $H: \Re^{n} \rightarrow \Re^{k}$ is a continuous map with $H(0)=0$. Moreover, there exists a constant $R \geqslant 0$ and a function $p \in K_{\infty}$ such that $|x| \leqslant R+p(|H(x)|)$.

(H4) The function $h: \Re^{n} \times U \rightarrow(0, r]$ is a positive, continuous and bounded function.

Hypotheses (H2) and (H4) are standard continuity and local-boundedness assumptions. Hypothesis (H3) restricts the class of output mappings that are allowed to be studied in the present framework. However, note that the important case of the identity output $(H(x) \equiv x)$ is allowed.

The following theorem is an immediate consequence of the results presented in [44].

Theorem 3.1

Consider the control system (4) under hypotheses $(\mathrm{H} 1-\mathrm{H} 4)$ and let $\phi\left(t, t_{0}, x_{0} ; v, d, \tilde{d}\right)$ denote the solution of (4) with initial condition $x\left(t_{0}\right)=x_{0}$ corresponding to input $(d, \tilde{d}, v) \in M(D) \times M\left(\Re^{+}\right) \times$ $M(U)$. Then

(i) system (4) has the 'boundedness-implies-continuation' (BIC) property, i.e. for each $\left(t_{0}, x_{0}, v, d, \tilde{d}\right) \in \mathfrak{R}^{+} \times \mathfrak{R}^{n} \times M(U) \times M(D) \times M\left(\Re^{+}\right)$, there exists $t_{\max } \in\left(t_{0},+\infty\right] \quad$ the maximal existence time of the solution) such that the solution $\phi\left(t, t_{0}, x_{0} ; v, d, \tilde{d}\right)$ of (4) exists for all $t \in\left[t_{0}, t_{\max }\right)$. In addition, if $t_{\max }<+\infty$ then for every $C>0$ there exists $t \in\left[t_{0}, t_{\max }\right)$ with $\left|\phi\left(t, t_{0}, x_{0} ; v, d, \tilde{d}\right)\right|>C$.

(ii) $0 \in \mathfrak{R}^{n}$ is a robust equilibrium point from the input $(v, \tilde{d}) \in M(U) \times M\left(\mathfrak{R}^{+}\right)$, i.e. for every $\varepsilon>0, T \in \mathfrak{R}^{+}$there exists $\delta:=\delta(\varepsilon, T)>0$ such that for all $\left(t_{0}, x_{0}, v, \tilde{d}\right) \in[0, T] \times$ $\Re^{n} \times M(U) \times M\left(\Re^{+}\right)$, with $\left|x_{0}\right|+\sup _{t \geqslant 0}|v(t)|+\sup _{t \geqslant 0} \tilde{d}(t)<\delta$ it holds that the solution $\phi\left(t, t_{0}, x_{0} ; v, d, \tilde{d}\right)$ of (4) exists for all $t \in\left[t_{0}, t_{0}+T\right]$ and $d \in M(D)$ and

$$
\sup \left\{\left|\phi\left(t, t_{0}, x_{0} ; v, d, \tilde{d}\right)\right| ; d \in M(D), t \in\left[t_{0}, t_{0}+T\right], t_{0} \in[0, T]\right\}<\varepsilon
$$

(iii) system (4) is autonomous, i.e. for each $\left(t_{0}, x_{0}, v, d, \tilde{d}\right) \in \mathfrak{R}^{+} \times \mathfrak{R}^{n} \times M(U) \times M(D) \times$ $M\left(\Re^{+}\right), t \geqslant t_{0}$ and for each $\theta \in\left(-\infty, t_{0}\right]$ it holds that $\phi\left(t, t_{0}, x_{0}, v, d, \tilde{d}\right)=\phi\left(t-\theta, t_{0}-\theta, x_{0}\right.$, $\left.P_{\theta} v, P_{\theta} d, P_{\theta} \tilde{d}\right)$, where $\left(P_{\theta} v\right)(t)=v(t+\theta),\left(P_{\theta} \tilde{d}\right)(t)=\tilde{d}(t+\theta)$ and $\left(P_{\theta} d\right)(t)=d(t+\theta)$ for all $t+\theta \geqslant 0$.

A few comments about Theorem 3.1: Theorem 3.1 guarantees that the solution of system (4) can be continued as long as the solution remains bounded (this is exactly the BIC property). Therefore, the only case that the solution cannot be continued is the so-called 'finite escape time' phenomenon (i.e. 'blow up' of the solution). As remarked in [47] the notion of the robust equilibrium point coincides with the usual notion of an equilibrium point for systems with continuous dependence on the initial data. However, for sampled-data systems, the property of continuous dependence on the initial data is very restrictive and closed-loop control systems with discontinuous sampled-data feedback stabilizers do not satisfy this property. Consequently, the weaker property of a robust equilibrium point is useful in this case and Theorem 3.1 guarantees that this particular property holds (see also the discussion in [44]). Finally, the fact that system (4) is autonomous allows us to assume that $t_{0}=0$. Theorem 3.1 is an immediate consequence of Propositions 3.5 and 3.7 in 
[44] (in fact in [44] the results are shown for a wider class of sampled-data systems including time-varying sampled-data systems with impulsive behavior).

We next present the notions of robust forward completeness and UIOS property for systems of the form (4) under hypotheses (H1-H4).

\section{Definition 3.2}

Consider the control system (4) under hypotheses (H1-H4) and let $\phi\left(t, x_{0} ; v, d, \tilde{d}\right)$ denote the solution of (4) with initial condition $x(0)=x_{0}$ corresponding to input $(d, \tilde{d}, v) \in M(D) \times M\left(\Re^{+}\right) \times$ $M(U)$. We say that (4) is robustly forward complete $(R F C)$ from the input $(v, \tilde{d}) \in M(U) \times M\left(\Re^{+}\right)$, if for every $R \geqslant 0, T \geqslant 0$, it holds that

$$
\sup \left\{\left|\phi\left(t, x_{0} ; v, d, \tilde{d}\right)\right| ; v \in M\left(B_{U}[0, R]\right), \tilde{d} \in M([0, R]), t \in[0, T],\left|x_{0}\right| \leqslant R, d \in M(D)\right\}<+\infty
$$

The property of robust forward completeness in conjunction with the BIC property guarantees the existence of the solutions for all $t \geqslant 0$. However, the reader should be aware that robust forward completeness does not guarantee a bound for the state for all $t \geqslant 0$ (see the discussion in [44, 47]).

The following definition is parallel to the corresponding notions used for finite-dimensional control systems described by ordinary differential equations (see [34-38]).

\section{Definition 3.3}

Consider the control system (4) under hypotheses $(\mathrm{H} 1-\mathrm{H} 4)$ and let $\phi\left(t, x_{0} ; v, d, \tilde{d}\right)$ denote the solution of (4) with initial condition $x(0)=x_{0}$ corresponding to input $(d, \tilde{d}, v) \in M(D) \times M\left(\Re^{+}\right) \times$ $M(U)$. Suppose that (4) is RFC from the input $(v, \tilde{d}) \in M(U) \times M\left(\Re^{+}\right)$and suppose that there exist functions $\sigma \in K L, \gamma \in \mathscr{N}$ such that the following estimate holds for all $\left(x_{0}, v, d, \tilde{d}\right) \in \Re^{n} \times$ $M(U) \times M(D) \times M\left(\Re^{+}\right)$and $t \geqslant 0$ :

$$
\left|H\left(\phi\left(t, x_{0} ; v, d, \tilde{d}\right)\right)\right| \leqslant \sigma\left(\left|x_{0}\right|, t\right)+\sup _{0 \leqslant \tau \leqslant t} \gamma(|v(\tau)|)
$$

Then we say that (4) satisfies the UIOS property with gain $\gamma \in \mathscr{N}$ from the input $v \in M(U)$ and zero gain from the input $\tilde{d} \in M\left(\Re^{+}\right)$. Particularly, if $H(x):=x$, then we say that (4) satisfies the UISS property with gain $\gamma \in \mathscr{N}$ from the input $v \in M(U)$ and zero gain from the input $\tilde{d} \in M\left(\Re^{+}\right)$.

For the proof of our main results we will need the following technical small-gain lemma. It is a direct corollary of Theorem 1 in [48] and is closely related to Lemma A.1 in [49].

\section{Lemma 3.4}

For every $\sigma \in K L$ and $a \in K$ with $a(s)<s$ for all $s>0$, there exists $\tilde{\sigma} \in K L$ with the following property: if $y:\left[t_{0}, t_{1}\right) \rightarrow \mathfrak{R}^{+}, u: \mathfrak{R}^{+} \rightarrow \mathfrak{R}^{+}$are locally bounded functions and $M \geqslant 0$ a constant such that the following inequality holds for all $\xi \in\left[t_{0}, t_{1}\right)$

$$
y(t) \leqslant \max \left\{\sigma(M, t-\xi) ; a\left(\sup _{\xi \leqslant \tau \leqslant t} y(\tau)\right) ; u(t)\right\} \quad \forall t \in\left[\xi, t_{1}\right)
$$

then the following estimate holds for all $t \in\left[t_{0}, t_{1}\right)$ :

$$
y(t) \leqslant \max \left\{\tilde{\sigma}\left(M, t-t_{0}\right) ; \sup _{t_{0} \leqslant \tau \leqslant t} u(\tau)\right\} \quad \forall t \in\left[t_{0}, t_{1}\right)
$$


Finally, we end this section by presenting the following comparison lemma, which provides a sharp estimate of the evolution of Lyapunov functions (compare the obtained estimate with Theorem 5.2, p. 218 in [45]). Its proof can be found in Appendix A. Note that a similar result with the following lemma is contained in [50].

\section{Lemma 3.5}

For each positive-definite continuous function $\rho: \mathfrak{R}^{+} \rightarrow \mathfrak{R}^{+}$there exists a function $\sigma$ of class $K L$, with $\sigma(s, 0)=s$ for all $s \geqslant 0$ with the following property: if $y:\left[t_{0}, t_{1}\right] \rightarrow \mathfrak{R}^{+}$is an absolutely continuous function, $u: \Re^{+} \rightarrow \Re^{+}$is a locally bounded mapping and $I \subset\left[t_{0}, t_{1}\right]$ a set of Lebesgue measure zero such that $\dot{y}(t)$ is defined on $\left[t_{0}, t_{1}\right] \backslash I$ and such that the following implication holds for all $t \in\left[t_{0}, t_{1}\right] \backslash I$ :

$$
y(t) \geqslant u(t) \Rightarrow \dot{y}(t) \leqslant-\rho(y(t))
$$

then the following estimate holds for all $t \in\left[t_{0}, t_{1}\right]$ :

$$
y(t) \leqslant \max \left\{\sigma\left(y\left(t_{0}\right), t-t_{0}\right), \sup _{t_{0} \leqslant s \leqslant t} \sigma(u(s), t-s)\right\}
$$

\section{MAIN RESULTS}

The main result of the present work is the following theorem, which provides sufficient conditions for the UIOS property for system (4) with gain $\gamma \in \mathscr{N}$ from the input $u \in M(U)$ and zero gain from the input $\tilde{d} \in M\left(\Re^{+}\right)$. The conditions are expressed by means of a vector Lyapunov function. It utilizes the method of Razumikhin functions (see [30-32]) for stability analysis of time-delay systems, together with recent developments in the theory of vector Lyapunov functions (see [33]).

\section{Theorem 4.1 (vector Lyapunov function characterization of UIOS)}

Consider system (4) under hypotheses $(\mathrm{H} 1-\mathrm{H} 4)$ and suppose that there exists a family of functions $V_{i} \in C^{1}\left(\Re^{n} ; \Re^{+}\right)(i=1, \ldots, k)$, functions $a_{1}, a_{2} \in K_{\infty}, a, \zeta \in \mathscr{N}$ with $a(s)<s$ for all $s>0$ and a family of positive-definite functions $\rho_{i} \in C^{0}\left(\Re^{+} ; \Re^{+}\right)(i=1, \ldots, k)$, such that

$$
a_{1}(|H(x)|) \leqslant \max _{i=1, \ldots, k} V_{i}(x) \leqslant a_{2}(|x|) \quad \forall x \in \Re^{n}
$$

and for every $i=1, \ldots, k$ and $\left(x, v, v_{0}\right) \in \Re^{n} \times U \times U$ the following implication holds:

$$
\begin{aligned}
& \text { 'If } \max \left\{\zeta(|v|), \zeta\left(\left|v_{0}\right|\right), \max _{j=1, \ldots, k} a\left(V_{j}(x)\right)\right\} \leqslant V_{i}(x) \text { and } x_{0} \in A_{i}\left(h\left(x_{0}, v_{0}\right), x\right) \text {, then } \\
& \quad \sup \left\{\nabla V_{i}(x) f\left(x, x_{0}, d, v, v_{0}\right): d \in D\right\} \leqslant-\rho_{i}\left(V_{i}(x)\right) \text { ' }
\end{aligned}
$$

where the family of set-valued maps $\Re^{+} \times \Re^{n} \ni(T, x) \rightarrow A_{i}(T, x) \subseteq \Re^{n} \ni(i=1, \ldots, k)$ is defined by

$$
A_{i}(T, x)=\bigcup_{0 \leqslant s \leqslant T}\left\{\begin{array}{l}
x_{0} \in \Re^{n}: \exists(d, v) \in M(D) \times M(U) \text { with } \phi\left(s, x_{0} ; d, v\right)=x \\
\zeta(|v(t)|) \leqslant V_{i}(x), a\left(V_{j}\left(\phi\left(t, x_{0} ; d, v\right)\right) \leqslant V_{i}(x)\right. \\
\text { for all } t \in[0, s] \text { and } j=1, \ldots, k
\end{array}\right\}
$$


and $\phi\left(t, x_{0} ; d, v\right)$ denotes the solution of $\dot{x}(t)=f\left(x(t), x_{0}, d(t), v(t), v(0)\right)$ with initial condition $x(0)=x_{0}$ corresponding to $(d, v) \in M(D) \times M(U)$.

Then (4) satisfies the UIOS property with gain $\gamma=a_{1}^{-1} \circ \zeta \in \mathscr{N}$ from the input $v \in M(U)$ and zero gain from the input $\tilde{d} \in M\left(\Re^{+}\right)$.

\section{Remark 4.2}

The interpretation of the family of set-valued maps $\mathfrak{R}^{+} \times \mathfrak{R}^{n} \ni(T, x) \rightarrow A_{i}(T, x) \subseteq \Re^{n}(i=1, \ldots, k)$ defined in (17) is the following: each $A_{i}(T, x) \subseteq \Re^{n}$ is the set of all states $x_{0} \in \Re^{n}$ so that the solution of $\dot{x}(t)=f\left(x(t), x_{0}, d(t), v(t), v(0)\right)$ with initial condition $x(0)=x_{0}$ can be controlled to $x \in \Re^{n}$ in time $s$ less or equal than $T$ by means of appropriate inputs $(d, v) \in M(D) \times M(U)$ that satisfy $\zeta\left(\sup _{t \in[0, s]}|v(t)|\right) \leqslant V_{i}(x)$ and such that the trajectory of the solution satisfies the constraint $\sup _{t \in[0, s]} a\left(V_{j}(x(t))\right) \leqslant V_{i}(x)(j=1, \ldots, k)$.

In general it is very difficult to obtain an accurate description of the set-valued maps $\Re^{+} \times \Re^{n} \ni(T, x) \rightarrow A_{i}(T, x) \subseteq \Re^{n}$ defined by (17). However, if $a, \zeta \in K_{\infty}$, then for every $g \in$ $C^{1}\left(\Re^{n} ; \Re\right)$, we have

$$
A_{i}(T, x) \subseteq B_{i}^{g}(T, x)=\left\{x_{0} \in \Re^{n}:\left|g\left(x_{0}\right)-g(x)\right| \leqslant T b_{i}^{g}(x)\right\} \quad \forall(T, x) \in \Re^{+} \times \Re^{n}
$$

where

$$
\begin{aligned}
b_{i}^{g}(x) & :=\max \left\{\left|\nabla g(\xi) f\left(\xi, x_{0}, d, v, v_{0}\right)\right|: d \in D, \zeta\left(\max \left\{|v|,\left|v_{0}\right|\right\}\right)\right. \\
& \left.\leqslant V_{i}(x), a\left(\max \left\{V(\xi), V\left(x_{0}\right)\right\}\right) \leqslant V_{i}(x)\right\}<+\infty
\end{aligned}
$$

$a, \zeta \in \mathscr{N}, V_{i} \in C^{1}\left(\Re^{n} ; \Re^{+}\right)(i=1, \ldots, k)$ are the functions involved in hypotheses of Theorem 4.1 and $V(x)=\max _{i=1, \ldots, k} V_{i}(x)$. In order to justify the inclusion $A_{i}(T, x) \subseteq B_{i}^{g}(T, x)$, note that for all $\left(d, v, x_{0}\right) \in M(D) \times M(U) \times \Re^{n}$ it holds that

$$
g\left(\phi\left(s, x_{0} ; d, v\right)\right)-g\left(x_{0}\right)=\int_{0}^{s} \nabla g\left(\phi\left(t, x_{0} ; d, v\right)\right) f\left(\phi\left(t, x_{0} ; d, v\right), x_{0}, d(t), v(t), v(0)\right) \mathrm{d} t
$$

for all $s \geqslant 0$ for which $\phi\left(s, x_{0} ; d, v\right)$ exists (i.e. the solution of $\dot{x}(t)=f\left(x(t), x_{0}, d(t), v(t), v(0)\right)$ with initial condition $x(0)=x_{0}$ corresponding to $(d, v) \in M(D) \times M(U)$ exists for all $\left.t \in[0, s]\right)$. If $x_{0} \in A_{i}(T, x)$, by virtue of definition (17), there exists $s \in[0, h],(d, v) \in M(D) \times M(U)$ with $\zeta(|v(t)|) \leqslant V_{i}(x)$ for all $t \in[0, s]$ such that $a\left(V\left(\phi\left(t, x_{0} ; d, v\right)\right)\right) \leqslant V_{i}(x)$ for all $t \in[0, s]$ and $\phi\left(s, x_{0} ; d, v\right)=x$ (where $V(x)=\max _{i=1, \ldots, k} V_{i}(x)$ ). Clearly, the previous equality implies that if $x_{0} \in A_{i}(T, x)$, then there exists $s \in[0, h]$ such that

$$
\left|g(x)-g\left(x_{0}\right)\right| \leqslant s b_{i}^{g}(x) \leqslant T b_{i}^{g}(x)
$$

which shows that $x_{0} \in B_{i}^{g}(T, x)$.

Thus, in order to establish the UIOS property for (4), without knowledge of the exact solution map $\phi$, we must first select appropriate functions $g_{i} \in C^{1}\left(\Re^{n} ; \Re\right)(i=1, \ldots, k)$ and show that the following (more demanding) inequalities hold:

$$
\begin{aligned}
& \nabla V_{i}(x) f\left(x, x_{0}, d, v, v_{0}\right) \leqslant-\rho_{i}\left(V_{i}(x)\right) \quad \forall\left(x, d, v, v_{0}\right) \in \Re^{n} \times D \times U \times U \\
& \quad \text { with } \max \left\{\zeta(|v|), \zeta\left(\left|v_{0}\right|\right)\right\} \leqslant V_{i}(x) \text { and } x_{0} \in B_{i}^{g_{i}}\left(h\left(x_{0}, v_{0}\right), x\right)(i=1, \ldots, k)
\end{aligned}
$$

instead of (16). The examples of the following section illustrate the use of Theorem 4.1 in conjunction with (18). 
Since there is no guarantee that $B_{i}^{g}(T, x)$ approximates with sufficient accuracy the set $A_{i}(T, x)$ for an arbitrary function $g \in C^{1}\left(\Re^{n} ; \Re\right)$, it should be emphasized that (in practice) many different functions $g \in C^{1}\left(\Re^{n} ; \Re\right)$ may be tested. If the functions $g \in C^{1}\left(\Re^{n} ; \Re\right)$ belong to a set of functions $G \subset C^{1}\left(\Re^{n} ; \Re\right)$, then the (generally less conservative) set-valued map $\bigcap_{g \in G} B_{i}^{g}(T, x)$ can be used in place of $B_{i}^{g}(T, x)$. Note that the inclusion $A_{i}(T, x) \subseteq \bigcap_{g \in G} B_{i}^{g}(T, x)$ holds for every set of functions $G \subset C^{1}\left(\Re^{n} ; \Re\right)$.

\section{Proof of Theorem 4.1}

Consider a solution $x(t)$ of (4) under hypotheses $(\mathrm{H} 1-\mathrm{H} 4)$ corresponding to arbitrary $(v, d, \tilde{d}) \in$ $M(U) \times M(D) \times M\left(\Re^{+}\right)$with initial condition $x(0)=x_{0} \in \Re^{n}$. By virtue of Theorem 3.1, there exists a maximal existence time for the solution denoted by $t_{\max } \leqslant+\infty$. Let $V_{i}(t)=$ $V_{i}(x(t)), i=1, \ldots, k$, absolutely continuous functions on $\left[0, t_{\max }\right)$. Moreover, let $V(t):=$ $\max _{i=1, \ldots, k} V_{i}(t), \pi:=\left\{\tau_{0}, \tau_{1}, \ldots\right\}$ the set of sampling times (which may be finite if $t_{\max }<+\infty$ ) and $p(t):=\max \{\tau \in \pi: \tau \leqslant t\}, q(t):=\min \{\tau \in \pi: \tau \geqslant t\}$. Let $I \subset\left[0, t_{\max }\right)$ be the zero Lebesgue measure set, where $x(t)$ is not differentiable or where $\dot{x}(t) \neq f\left(x(t), x\left(\tau_{i}\right), d(t), v(t), v\left(\tau_{i}\right)\right)$. Clearly, we have $x(t)=\phi\left(t-p(t), x(p(t)) ; P_{t} d, P_{t} v\right)$ for all $t \in\left[0, t_{\max }\right)$, where $\left(P_{t} v\right)(s)=v(p(t)+s)$, $\left(P_{t} d\right)(s)=d(p(t)+s), s \geqslant 0$. Next, we show that the following implication holds for $t \in\left[0, t_{\max }\right) \backslash I$ and $i=1, \ldots, k$ :

$$
V_{i}(t) \geqslant \max \left\{\sup _{p(t) \leqslant s \leqslant t} \zeta(|v(s)|), \sup _{p(t) \leqslant s \leqslant t} a(V(s))\right\} \Rightarrow \dot{V}_{i}(t) \leqslant-\rho_{i}\left(V_{i}(t)\right)
$$

In order to prove implication (19) let $t \in\left[0, t_{\max }\right) \backslash I, i=1, \ldots, k, \tau=p(t)$ and suppose that

$$
V_{i}(t) \geqslant \max \left\{\sup _{p(t) \leqslant s \leqslant t} \zeta(|v(s)|), \sup _{p(t) \leqslant s \leqslant t} a(V(s))\right\}
$$

By virtue of the semigroup property for the previous inequality, it implies that $\zeta(|v(\tau+s)|)=$ $\zeta\left(\left|\left(P_{t} v\right)(s)\right|\right) \leqslant V_{i}(x(t)), a\left(V_{j}\left(\phi\left(s, x(\tau) ; P_{t} d, P_{t} v\right)\right)\right) \leqslant V_{i}(x(t))$ for all $s \in[0, t-\tau]$ and $j=1, \ldots, k$. In this case, by virtue of definition (17) and the fact that $t-\tau \leqslant h(x(\tau), v(\tau))$, it follows that $x(\tau) \in A_{i}(h(x(\tau), v(\tau)), x(t))$. Since $\dot{x}(t)=f(x(t), x(\tau), d(t), v(t), v(\tau))$, we conclude from (16) that $\dot{V}_{i}(t) \leqslant-\rho_{i}\left(V_{i}(t)\right)$.

Lemma 3.5 implies that there exists a family of continuous function $\sigma_{i}$ of class $K L(i=1, \ldots, k)$, with $\sigma_{i}(s, 0)=s$ for all $s \geqslant 0$ (which is independent of $\left(x_{0}, v, d, \tilde{d}\right) \in \Re^{n} \times M(U) \times M(D) \times M\left(\Re^{+}\right)$) such that for all $\xi \in\left[0, t_{\max }\right), t \in\left[\xi, t_{\max }\right)$ and $i=1, \ldots, k$ we have

$$
\begin{aligned}
V_{i}(t) \leqslant & \max \left\{\sigma_{i}\left(V_{i}(\xi), t-\xi\right) ; \sup _{\xi \leqslant \tau \leqslant t} \sigma_{i}\left(a\left(\sup _{p(\tau) \leqslant s \leqslant \tau} V(s)\right), t-\tau\right) ;\right. \\
& \left.\sup _{\xi \leqslant \tau \leqslant t} \sigma_{i}\left(\zeta\left(\sup _{p(\tau) \leqslant s \leqslant \tau}|v(s)|\right), t-\tau\right)\right\}
\end{aligned}
$$

Let $\sigma(s, t):=\max _{i=1, \ldots, k} \sigma_{i}(s, t)$, which is a function of class $K L$ that satisfies $\sigma(s, 0)=s$ for all $s \geqslant 0$. An immediate consequence of the previous definition, estimate (20) with $\xi=0$ and the fact 
that $\sigma_{i}(s, 0)=s$ for all $s \geqslant 0(i=1, \ldots, k)$, is the following estimate, which holds for all $t \in\left[0, t_{\max }\right)$ :

$$
V(t) \leqslant \max \left\{\sigma(V(0), t) ; a\left(\sup _{0 \leqslant s \leqslant t} V(s)\right) ; \sup _{0 \leqslant s \leqslant t} \zeta(|v(s)|)\right\}
$$

Inequality (21) and the fact that $\sigma(s, 0)=s$ for all $s \geqslant 0$ imply the following inequality:

$$
\sup _{0 \leqslant s \leqslant t} V(s) \leqslant \max \left\{V(0) ; a\left(\sup _{0 \leqslant s \leqslant t} V(s)\right) ; \sup _{0 \leqslant s \leqslant t} \zeta(|v(s)|)\right\} \quad \text { for all } t \in\left[0, t_{\max }\right)
$$

Making use of the fact that $a(s)<s$ for all $s>0$, we obtain from (22)

$$
V(t) \leqslant \max \left\{V(0) ; \sup _{0 \leqslant s \leqslant t} \zeta(|v(s)|)\right\} \quad \text { for all } t \in\left[0, t_{\max }\right)
$$

Clearly, inequality (23) implies that as long as the solution of (4) exists, $V(t)$ is bounded. Next, we show that $t_{\max }=+\infty$. The proof follows a standard contradiction argument. Suppose that $t_{\max }<+\infty$. The 'BIC' property for (4) (see Theorem 3.1) implies that for every $C>0$ there exists $t \in\left[0, t_{\max }\right)$ such that $|x(t)|>C$. Inequalities (15), (23) and hypothesis (H3) imply that for every $C>0$ there exists $t \in\left[0, t_{\max }\right)$ such that

$$
C<|x(t)| \leqslant R+p\left(a_{1}^{-1}\left(V(0)+\sup _{0 \leqslant s \leqslant t_{\max }} \zeta(|v(s)|)\right)\right)<+\infty
$$

Clearly, the previous inequality cannot hold for

$$
C \geqslant R+p\left(a_{1}^{-1}\left(V(0)+\sup _{0 \leqslant s \leqslant t_{\max }} \zeta(|v(s)|)\right)\right)
$$

(a contradiction). Thus, necessarily we must have $t_{\max }=+\infty$.

We conclude that estimate (20) holds for all $\xi \geqslant 0, t \geqslant \xi$ and $i=1, \ldots, k$. Similarly, estimate (23) holds for all $t \geqslant 0$.

An immediate consequence of estimate (20) with $\xi \in \pi$ (where $\pi:=\left\{\tau_{0}, \tau_{1}, \ldots\right\}$ is the set of sampling times), estimate (23), definitions $V(t):=\max _{i=1, \ldots, k} V_{i}(t), \sigma(s, t):=\max _{i=1, \ldots, k} \sigma_{i}(s, t)$ and the fact that $\sigma_{i}(s, 0)=s$ for all $s \geqslant 0(i=1, \ldots, k)$ is the following estimate, which holds for all $t \geqslant \xi, \xi \in \pi$ :

$$
V(t) \leqslant \max \left\{\sigma(V(0), t-\xi) ; a\left(\sup _{\xi \leqslant s \leqslant t} V(s)\right) ; \sup _{0 \leqslant s \leqslant t} \zeta(|v(s)|)\right\}
$$

We next show that the following estimate holds for all $\xi \geqslant 0, t \geqslant \xi$ :

$$
V(t) \leqslant \max \left\{\tilde{\sigma}(V(0), t-\xi) ; a\left(\sup _{\xi \leqslant s \leqslant t} V(s)\right) ; \sup _{0 \leqslant s \leqslant t} \zeta(|v(s)|)\right\}
$$

where $\tilde{\sigma}(s, t):=s \exp (r-t)$ for $t \in[0, r]$ and $\tilde{\sigma}(s, t):=\sigma(s, t-r)$ for $t>r, r>0$ being the upper bound for $h$. Since $\sigma(s, 0)=s$ for all $s \geqslant 0$, it follows that $\tilde{\sigma}$ is of class $K L$. 
First note that estimate (24) implies estimate (25) when $\xi \in \pi$. We continue by showing that estimate (25) holds for the case $\xi \notin \pi, \xi \geqslant 0$, as well. Let arbitrary $\xi \notin \pi, \xi \geqslant 0$. Since $\xi \notin \pi$, it follows that $p(\xi)<\xi<q(\xi)$ (where $p(t):=\max \{\tau \in \pi: \tau \leqslant t\}, q(t):=\min \{\tau \in \pi: \tau \geqslant t\}$ ). Note that by virtue of (23) and the previous definition of $\tilde{\sigma} \in K L$, it follows that (25) holds for all $t \leqslant \xi+r$. Inequality (20) gives for all $t \geqslant \xi+r \geqslant q(\xi)$ and $i=1, \ldots, k$ :

$$
V_{i}(t) \leqslant \max \left\{\begin{array}{l}
\sigma_{i}\left(V_{i}(\xi), t-\xi\right) ; \sup _{\xi \leqslant \tau<q(\xi)} \sigma_{i}\left(a\left(\sup _{p(\tau) \leqslant s \leqslant \tau} V(s)\right), t-\tau\right) \\
\sup _{q(\xi) \leqslant \tau \leqslant t} \sigma_{i}\left(a\left(\sup _{p(\tau) \leqslant s \leqslant \tau} V(s)\right), t-\tau\right) ; \sup _{p(\xi) \leqslant s \leqslant t} \zeta(|v(s)|)
\end{array}\right\}
$$

Note that if $\xi \leqslant \tau<q(\xi)$, then $p(\tau)=p(\xi)$. Moreover, if $\tau \geqslant q(\xi)$, then $p(\tau) \geqslant \xi$. Thus, by making use of the facts that $a(s) \leqslant s$ for all $s \geqslant 0$ and $q(\xi) \leqslant \xi+r$, we obtain from (26)

$$
V_{i}(t) \leqslant \max \left\{\begin{array}{l}
\sigma_{i}\left(V_{i}(\xi), t-\xi\right) ; \sup _{\xi \leqslant \tau<\xi+r} \sigma_{i}\left(\sup _{p(\xi) \leqslant s \leqslant \tau} V(s), t-\tau\right) \\
a\left(\sup _{\xi \leqslant s \leqslant t} V(s)\right) ; \sup _{p(\xi) \leqslant s \leqslant t} \zeta(|v(s)|)
\end{array}\right\} \forall t \geqslant \xi+r, i=1, \ldots, k
$$

Inequality (23) in conjunction with inequality (27) and the fact that $\sigma_{i}(s, 0)=s$ for all $s \geqslant 0(i=$ $1, \ldots, k)$ give

$$
V_{i}(t) \leqslant \max \left\{\sigma_{i}(V(0), t-\xi-r) ; a\left(\sup _{\xi \leqslant s \leqslant t} V(s)\right) ; \sup _{0 \leqslant s \leqslant t} \zeta(|v(s)|)\right\} \quad \forall t \geqslant \xi+r, \quad i=1, \ldots, k
$$

Since $\sigma(s, t):=\max _{i=1, \ldots, k} \sigma_{i}(s, t)$ and $V(t)=\max _{i=1, \ldots, k} V_{i}(t)$, we obtain from (28)

$$
V(t) \leqslant \max \left\{\sigma(V(0), t-\xi-r) ; a\left(\sup _{\xi \leqslant s \leqslant t} V(s)\right) ; \sup _{0 \leqslant s \leqslant t} \zeta(|v(s)|)\right\} \quad \forall t \geqslant \xi+r
$$

We conclude from (29) and definition of $\tilde{\sigma} \in K L$ that (25) holds for that case $t \geqslant \xi+r$, as well.

Lemma 3.4 in conjunction with inequality (25) implies the existence of $\bar{\sigma} \in K L$ such that

$$
V(t) \leqslant \max \left\{\bar{\sigma}(V(0), t) ; \sup _{0 \leqslant s \leqslant t} \zeta(|v(s)|)\right\}
$$

Clearly, estimate (30) in conjunction with inequality (15) shows that (4) satisfies the UIOS property with gain $\gamma=a_{1}^{-1} \circ \zeta \in \mathscr{N}$ from the input $v \in M(U)$ and zero gain from the input $\tilde{d} \in M\left(\Re^{+}\right)$. The proof is complete.

The following corollary is an immediate consequence of Theorem 4.1 and the fact that for every positive-definite and radially unbounded function $V \in C^{1}\left(\Re^{n} ; \Re^{+}\right)$and for every positivedefinite function $W \in C^{0}\left(\Re^{n} ; \Re^{+}\right)$, there exists a positive-definite function $\rho \in C^{0}\left(\Re^{+} ; \Re^{+}\right)$such that $\rho(V(x)) \leqslant W(x)$, for all $x \in \Re^{n}$. 
Corollary 4.3 (single Lyapunov function characterization of UISS)

Consider system (4) under hypotheses $(\mathrm{H} 1-\mathrm{H} 4)$ and suppose that there exists a function $V \in$ $C^{1}\left(\Re^{n} ; \Re^{+}\right)$, functions $a_{1}, a_{2} \in K_{\infty}, a, \zeta \in \mathscr{N}$ with $a(s)<s$ for all $s>0$ and a positive-definite mapping $W \in C^{0}\left(\Re^{n} ; \Re^{+}\right)$, such that the following inequalities hold:

$$
a_{1}(|x|) \leqslant V(x) \leqslant a_{2}(|x|) \quad \forall x \in \Re^{n}
$$

$$
\nabla V(x) f\left(x, x_{0}, d, v, v_{0}\right) \leqslant-W(x) \quad \forall\left(x, d, v, v_{0}\right) \in \Re^{n} \times D \times U \times U
$$

$$
\text { with } \max \left\{\zeta(|v|), \zeta\left(\left|v_{0}\right|\right)\right\} \leqslant V(x) \text { and } x_{0} \in A\left(h\left(x_{0}, v_{0}\right), x\right)
$$

where the set-valued map $\mathfrak{R}^{+} \times \mathfrak{R}^{n} \ni(T, x) \rightarrow A(T, x) \subseteq \mathfrak{R}^{n}$ is defined by

$$
A(T, x)=\bigcup_{0 \leqslant s \leqslant T}\left\{\begin{array}{l}
x_{0} \in \Re^{n}: \exists(d, v) \in M(D) \times M(U) \text { with } \phi\left(s, x_{0} ; d, v\right)=x \\
\zeta(|v(t)|) \leqslant V(x) \text { and } a\left(V\left(\phi\left(t, x_{0} ; d, v\right)\right)\right) \leqslant V(x), \text { for all } t \in[0, s]
\end{array}\right\}
$$

and $\phi\left(t, x_{0} ; d, v\right)$ denotes the solution of $\dot{x}(t)=f\left(x(t), x_{0}, d(t), v(t), v(0)\right)$ with initial condition $x(0)=x_{0}$ corresponding to $(d, v) \in M(D) \times M(U)$.

Then (4) satisfies the UISS property with gain $\gamma=a_{1}^{-1} \circ \zeta \in \mathscr{N}$ from the input $v \in M(U)$ and zero gain from the input $\tilde{d} \in M\left(\Re^{+}\right)$.

It should be noted that in practice (where the solution map is rarely known), Corollary 4.3 is used in conjunction with Remark 4.2.

\section{ILLUSTRATING EXAMPLES}

The following example illustrates the use of Theorem 4.1 and Corollary 4.3. Moreover, it shows that the use of vector Lyapunov functions provides flexibility, which is not easily obtained with the use of a single Lyapunov function.

\section{Example 5.1}

Consider the planar control system:

$$
\begin{aligned}
\dot{x}_{1} & =-2 x_{1}-d_{1} x_{1}^{3}+x_{2}, \quad \dot{x}_{2}=d_{2} x_{2}^{2}-x_{2}^{3}+u \\
x & =\left(x_{1}, x_{2}\right)^{\prime} \in \Re, \quad d=\left(d_{1}, d_{2}\right) \in D:=[\delta, \Delta] \times[-1,1], \quad u \in \Re
\end{aligned}
$$

We consider the closed-loop system (34) with the sampled feedback $u(t)=-2 x_{2}\left(\tau_{i}\right)+v(t), t \in$ $\left[\tau_{i}, \tau_{i+1}\right)$ and positive sampling rate, i.e. we consider the system:

$$
\begin{aligned}
\dot{x}_{1}(t) & =-2 x_{1}(t)-d_{1}(t) x_{1}^{3}(t)+x_{2}(t), \quad t \in\left[\tau_{i}, \tau_{i+1}\right) \\
\dot{x}_{2}(t) & =d_{2}(t) x_{2}^{2}(t)-x_{2}^{3}(t)-2 x_{2}\left(\tau_{i}\right)+v(t), \quad t \in\left[\tau_{i}, \tau_{i+1}\right) \\
\tau_{0} & =t_{0}, \quad \tau_{i+1}=\tau_{i}+r \exp \left(-\tilde{d}\left(\tau_{i}\right)\right) \\
x(t) & =\left(x_{1}(t), x_{2}(t)\right)^{\prime} \in \mathfrak{R}^{2} \\
\left(d_{1}(t), d_{2}(t), \tilde{d}(t), v(t)\right) & \in[\delta, \Delta] \times[-1,1] \times \mathfrak{R}^{+} \times \Re
\end{aligned}
$$


It should be noted that the feedback law $u(t)=-2 x_{2}\left(\tau_{i}\right)$ is obtained from the discretization of the globally stabilizing continuous-time feedback $u(t)=-2 x_{2}(t)$ (emulation procedure). This example was selected so that

(i) results concerning globally Lipschitz systems (see [17]) cannot be applied since system (35) is not globally Lipschitz;

(ii) results concerning homogeneous systems (see [16]) cannot be applied since system (35) is not homogeneous;

(iii) if the results contained in [20,21,23] were applied to system (35) then they would lead to local stability properties;

(iv) if the results contained in [4,9-11] were applied to system (35), then they would lead to semiglobal practical stability properties.

We next show that there exist $r, K>0$ and $\delta, \Delta \in \mathfrak{R}^{+}$with $\delta \leqslant \Delta$ such that system (35) satisfies the UISS property with gain $\gamma(s)=K s \in \mathscr{N}$ from the input $v \in M(\Re)$ and zero gain from the input $\tilde{d} \in M\left(\Re^{+}\right)$. In order to show the advantages of the use of vector Lyapunov functions, we perform the analysis using a single and a vector Lyapunov function.

Note that for every $\delta, \Delta \in \mathfrak{R}^{+}$with $\delta \leqslant \Delta$ and $r>0$, system (35) with output $H(x)=x$ satisfies hypotheses (H1-H4).

\subsection{First method: single Lyapunov function}

Consider the function:

$$
V(x)=\frac{1}{2} x_{1}^{2}+\frac{1}{2} x_{2}^{2}
$$

Clearly, the function $V \in C^{1}\left(\Re^{2} ; \mathfrak{R}^{+}\right)$defined by (36) satisfies (31) with $a_{1}(s)=a_{2}(s)=\frac{1}{2} s^{2}$. Moreover, by virtue of (35), definition (36), and the inequalities $x_{1} x_{2} \leqslant \frac{1}{2} x_{1}^{2}+\frac{1}{2} x_{2}^{2},\left|x_{2}^{3}\right| \leqslant \frac{1}{2} x_{2}^{2}+\frac{1}{2} x_{2}^{4}$, we obtain for all $\left(x_{1}, x_{2}, x_{2,0}, d_{1}, d_{2}, v\right) \in \mathfrak{R}^{3} \times[\delta, \Delta] \times[-1,1] \times \Re$

$$
\begin{aligned}
\nabla V(x)\left[\begin{array}{c}
-2 x_{1}-d_{1} x_{1}^{3}+x_{2} \\
d_{2} x_{2}^{2}-x_{2}^{3}-2 x_{2,0}+v
\end{array}\right] & \leqslant-2 x_{1}^{2}-\delta x_{1}^{4}+x_{1} x_{2}+d_{2} x_{2}^{3}-x_{2}^{4}-2 x_{2} x_{2,0}+x_{2} v \\
& \leqslant-\frac{3}{2} x_{1}^{2}-\delta x_{1}^{4}-x_{2}^{2}-\frac{1}{2} x_{2}^{4}-2 x_{2}\left(x_{2,0}-x_{2}\right)+\left|x_{2} \| v\right|
\end{aligned}
$$

Let $g(x):=x_{2}, \zeta(s):=2 s^{2} \in \mathscr{N}$ and $a(s):=c^{-2} s \in \mathscr{N}$, where $c>1$. It follows from (35) and (36) that the mapping $b^{g}(x):=\max \left\{\left|\nabla g(\xi) f\left(\xi, x_{0}, d, v\right)\right|: d \in D, a\left(\max \left\{V(\xi), V\left(x_{0}\right)\right\}\right) \leqslant V(x), \zeta(|v|) \leqslant\right.$ $V(x)\}$ satisfies the following inequality for all $\left(x_{1}, x_{2}\right) \in \mathfrak{R}^{2}$ :

$$
b^{g}(x) \leqslant c^{2}\left(x_{1}^{2}+x_{2}^{2}\right)+c^{3}\left(x_{1}^{2}+x_{2}^{2}\right)^{3 / 2}+\left(2 c+\frac{1}{2}\right)\left(x_{1}^{2}+x_{2}^{2}\right)^{1 / 2}
$$

Indeed, note that $\left|\nabla g(\xi) f\left(\xi, x_{0}, d, v\right)\right|=\left|d_{2} \xi_{2}^{2}-\xi_{2}^{3}-2 x_{2,0}+v\right| \leqslant \xi_{2}^{2}+\left|\xi_{2}\right|^{3}+2\left|x_{2,0}\right|+|v|$. Since $a\left(\max \left\{V(\xi), V\left(x_{0}\right)\right\}\right) \leqslant V(x)$, we obtain $\left|\xi_{2}\right| \leqslant c|x|$ and $\left|x_{2,0}\right| \leqslant c|x|$. Furthermore, since $\zeta(|v|) \leqslant$ $V(x)$, we obtain $|v| \leqslant \frac{1}{2}|x|$. Combining all previous inequalities we obtain (38). 
Using Young's inequalities and the above inequality, we obtain for all $\left(x_{1}, x_{2}\right) \in \mathfrak{R}^{2}$

$$
\left|x_{2}\right| b^{g}(x) \leqslant \frac{5 c^{2}+1}{2} x_{2}^{2}+\left(c+\frac{1}{4}\right) x_{1}^{2}+\frac{5 c^{3}}{2} x_{1}^{4}+\frac{11 c^{3}}{4} x_{2}^{4}
$$

Inequality (39) follows from (38) and the inequalities

$$
\begin{aligned}
\left(2 c+\frac{1}{2}\right)\left|x_{2}\right|\left(x_{1}^{2}+x_{2}^{2}\right)^{1 / 2} & \leqslant\left(2 c+\frac{1}{2}\right) x_{2}^{2}+\left(c+\frac{1}{4}\right) x_{1}^{2} \\
c^{2}\left|x_{2}\right|\left(x_{1}^{2}+x_{2}^{2}\right) & \leqslant \frac{c^{2}}{2} x_{2}^{2}+c^{3} x_{1}^{4}+c^{3} x_{2}^{4} \\
\left|x_{2}\right|\left(x_{1}^{2}+x_{2}^{2}\right)^{3 / 2} & \leqslant \frac{1}{4} x_{2}^{4}+\frac{3}{4}\left(x_{1}^{2}+x_{2}^{2}\right)^{2} \leqslant \frac{7}{4} x_{2}^{4}+\frac{3}{2} x_{1}^{4}
\end{aligned}
$$

Since $A(h, x) \subseteq B^{g}(h, x)=\left\{x_{0} \in \mathfrak{R}^{2}:\left|g\left(x_{0}\right)-g(x)\right| \leqslant h b^{g}(x)\right\}=\left\{\left(x_{1,0}, x_{2,0}\right) \in \mathfrak{R}^{2}:\left|x_{2}-x_{2,0}\right| \leqslant\right.$ $\left.h b^{g}(x)\right\}$ for all $(h, x) \in \mathfrak{R}^{+} \times \mathfrak{R}^{2}$ (see Remark 4.2), it follows from (37), (39) and the inequality $|v| \leqslant \frac{1}{2}|x|$ (which implies $\left|x_{2}\left\|v\left|\leqslant \frac{1}{2}\right| x_{2}\right\| x\right| \leqslant \frac{1}{4} x_{2}^{2}+\frac{1}{4}|x|^{2}=\frac{1}{4} x_{1}^{2}+\frac{1}{2} x_{2}^{2}$ ) that the following inequality holds for all $(h, x, d) \in \mathfrak{R}^{+} \times \mathfrak{R}^{2} \times D, \zeta(|v|) \leqslant V(x)$ and $\left(x_{1,0}, x_{2,0}\right)^{\prime} \in A(h, x)$ :

$$
\begin{aligned}
\nabla V(x)\left[\begin{array}{c}
-2 x_{1}-d_{1} x_{1}^{3}+x_{2} \\
d_{2} x_{2}^{2}-x_{2}^{3}-2 x_{2,0}+v
\end{array}\right] \leqslant & -\frac{1}{4}(5-8 c h-2 h) x_{1}^{2}-\left(\delta-5 c^{3} h\right) x_{1}^{4} \\
& -\frac{1}{2}\left(1-10 c^{2} h-2 h\right) x_{2}^{2}-\frac{1}{2}\left(1-11 c^{3} h\right) x_{2}^{4}
\end{aligned}
$$

Inequality (40) guarantees that (32) holds if $\delta>0$ and if we define $h(x):=r>0$ with

$$
r<\frac{1}{10 c^{2}+2} \text { and } r \leqslant c^{-3} \min \left\{\frac{1}{11} ; \frac{\delta}{5}\right\}
$$

and $W(x)=\mu|x|^{2}$ for $\mu:=\frac{1}{2}\left(1-10 c^{2} r-2 r\right)$. We conclude from Corollary 4.3 that system (35) satisfies the UISS property with gain $\gamma(s)=K s \in \mathscr{N}$ (with $K=2$ ) from the input $v \in M(\Re)$ and zero gain from the input $\tilde{d} \in M\left(\Re^{+}\right)$.

\subsection{Second method: vector Lyapunov function}

Consider the functions:

$$
V_{1}(x)=\frac{1}{2} x_{1}^{2}, \quad V_{2}(x)=\frac{1}{2} x_{2}^{2}
$$

Clearly, the functions $V_{i} \in C^{1}\left(\Re^{2} ; \Re^{+}\right), i=1,2$ defined by (42) satisfy (15) with $H(x):=x, a_{1}(s)=$ $\frac{1}{4} s^{2}, a_{2}(s)=\frac{1}{2} s^{2}$ (an immediate consequence of the inequality $\frac{1}{2}(a+b) \leqslant \max \{a, b\} \leqslant a+b$, which holds for all $a, b \geqslant 0)$. Let $\zeta(s):=2 s^{2} \in \mathscr{N}$ and $a(s):=c^{-2} s \in \mathscr{N}$, where $c \in(1,2)$.

By virtue of (35) and definition (42), we obtain for all $\delta \geqslant 0,\left(x_{1}, x_{2}, d_{1}\right) \in \Re^{2} \times[\delta, \Delta]$ with $a\left(V_{2}(x)\right) \leqslant V_{1}(x)$ :

$$
\nabla V_{1}(x)\left(-2 x_{1}-d_{1} x_{1}^{3}+x_{2}\right) \leqslant-2 x_{1}^{2}-\delta x_{1}^{4}+x_{1} x_{2} \leqslant-(2-c) x_{1}^{2}=-2(2-c) V_{1}(x)
$$

The above inequality guarantees that (16) holds for $i=1$ with $\rho_{1}(s)=2(2-c) s$. 
Furthermore, we obtain from (35), definition (42), and the inequality $\left|x_{2}^{3}\right| \leqslant \frac{1}{2} x_{2}^{2}+\frac{1}{2} x_{2}^{4}$ for all $\left(x_{1}, x_{2}, d_{2}, v\right) \in \Re^{2} \times[-1,1] \times \Re$ with $\zeta(|v|) \leqslant V_{2}(x)$ (which implies $\left.|v| \leqslant \frac{1}{2}\left|x_{2}\right|\right)$ :

$$
\nabla V_{2}(x)\left(d_{2} x_{2}^{2}-x_{2}^{3}-2 x_{2,0}+v\right)=d_{2} x_{2}^{3}-x_{2}^{4}-2 x_{2} x_{2,0}+x_{2} v \leqslant-x_{2}^{2}-\frac{1}{2} x_{2}^{4}-2 x_{2}\left(x_{2,0}-x_{2}\right)
$$

Let $g(x):=x_{2}$. It follows from (35) and (42) that the mapping $b^{g}(x):=\max \left\{\mid \nabla g(\xi) f\left(\xi, x_{0}\right.\right.$, $\left.d, v) \mid: d \in D, a\left(\max \left\{V(\xi), V\left(x_{0}\right)\right\}\right) \leqslant V_{2}(x), \zeta(|v|) \leqslant V_{2}(x)\right\}$ satisfies the following inequality for all $\left(x_{1}, x_{2}\right) \in \Re^{2}$ :

$$
b^{g}(x) \leqslant c^{2} x_{2}^{2}+c^{3}\left|x_{2}\right|^{3}+\left(2 c+\frac{1}{2}\right)\left|x_{2}\right|
$$

Since $A(h, x) \subseteq B^{g}(h, x)=\left\{x_{0} \in \mathfrak{R}^{2}:\left|g\left(x_{0}\right)-g(x)\right| \leqslant h b^{g}(x)\right\}=\left\{\left(x_{1,0}, x_{2,0}\right) \in \mathfrak{R}^{2}:\left|x_{2}-x_{2,0}\right| \leqslant h b^{g}(x)\right\}$ for all $(h, x) \in \Re^{+} \times \mathfrak{R}^{2}$ (see Remark 4.2), it follows from (43), (44) and $c^{2}\left|x_{2}^{3}\right| \leqslant\left(c^{2} / 2\right) x_{2}^{2}+\left(c^{3} / 2\right) x_{2}^{4}$ that the following inequality holds for all $\left(x_{1}, x_{2}, d_{2}, v\right) \in \mathfrak{R}^{2} \times[-1,1] \times \Re$ with $\zeta(|v|) \leqslant V_{2}(x)$ and $\left(x_{1,0}, x_{2,0}\right)^{\prime} \in A_{2}(h, x)$ :

$$
\nabla V_{2}(x)\left(d_{2} x_{2}^{2}-x_{2}^{3}-2 x_{2,0}+v\right) \leqslant-\left(1-h-5 c^{2} h\right) x_{2}^{2}-\frac{1}{2}\left(1-6 c^{3} h\right) x_{2}^{4}
$$

Inequality (45) guarantees that (16) for $i=2$ holds if we define $h(x):=r>0$ with

$$
r \leqslant\left(6 c^{3}\right)^{-1}
$$

and $\rho_{2}(s)=\left(\left(1-r-5 c^{2} r\right) / 2\right) s$. We conclude from Theorem 4.1 that system (35) satisfies the UISS property with gain $\gamma(s)=K s \in \mathscr{N}$ (with $K=2 \sqrt{2}$ ) from the input $v \in M(\Re)$ and zero gain from the input $\tilde{d} \in M\left(\Re^{+}\right)$.

\subsection{Comparison of the two methods}

We are now ready to compare the two methods. It is clear from (41) and (46) that the vector Lyapunov function method is less conservative compared with the single Lyapunov method since

(i) it allows greater sampling periods;

(ii) we do not have to assume that $\delta>0$ (only $\delta \geqslant 0$ suffices and note that the size of $\delta$ does not affect the sampling rate; note also that the single Lyapunov method cannot allow $\delta=0$ since (41) must hold).

Of course, different Lyapunov functions may be used in order to obtain even less conservative results.

Example 5.1 shows that there exist nonlinear systems with no special characteristics (such as homogeneity or global Lipschitz properties), which can be globally asymptotically stabilized by means of sampled-data feedback with positive sampling rate. Working exactly as in the previous example with a vector Lyapunov function, we can establish that $0 \in \mathfrak{R}^{2}$ can be robustly globally asymptotically stabilized by linear feedback with zero-order hold and positive sampling rate for the system:

$$
\begin{aligned}
& \dot{x}_{1}=f_{1}\left(x_{1}, x_{2}, d\right) \\
& \dot{x}_{2}=f_{2}\left(x_{1}, x_{2}, d\right)+u \\
& x=\left(x_{1}, x_{2}\right)^{\prime} \in \mathfrak{R}^{2}, \quad d \in D, \quad u \in \mathfrak{R}
\end{aligned}
$$


where $D \subset \Re^{m}$ is a compact set, $f_{i}: \Re^{2} \times D \rightarrow \Re(i=1,2)$ are locally Lipschitz mappings with $f_{i}(0,0, d)=0$ for all $d \in D(i=1,2)$, under the following hypothesis:

(P) There exist constants $c>1, a \in \Re, L, \gamma>0$ such that

$$
\begin{aligned}
& \max \left\{x_{1} f_{1}\left(x_{1},-a x_{1}+\xi, d\right) ; \quad x_{1} \neq 0,|\xi| \leqslant c\left|x_{1}\right|, d \in D\right\}<0 \quad \text { for all } x_{1} \neq 0 \\
& |z| \max \left\{\left|f_{2}\left(x_{1},-a x_{1}+\xi, d\right)+a f_{1}\left(x_{1},-a x_{1}+\xi, d\right)\right| ; \max \left(\left|x_{1}\right|,|\xi|\right) \leqslant c|z|, d \in D\right\} \\
& \quad+L \max \left\{z f_{2}\left(x_{1},-a x_{1}+z, d\right)+a z f_{1}\left(x_{1},-a x_{1}+z, d\right) ;\left|x_{1}\right| \leqslant c|z|, d \in D\right\} \\
& \leqslant \gamma z^{2} \quad \text { for all } z \in \Re
\end{aligned}
$$

Hypothesis $(\mathrm{P})$ reminds a 'sector condition' imposed on the nonlinear functions $f_{1}, f_{2}$. It guarantees that the graphs of the nonlinear functions $f_{1}, f_{2}$ are located into appropriate conic sections of the plane.

Indeed, under hypothesis (P) we may conclude that there exist constants $r, R>0$ such that the following system:

$$
\begin{aligned}
\dot{x}_{1}(t) & =f_{1}\left(x_{1}(t), x_{2}(t), d(t)\right), \quad t \in\left[\tau_{i}, \tau_{i+1}\right) \\
\dot{x}_{2}(t) & =f_{2}\left(x_{1}(t), x_{2}(t), d(t)\right)-R\left(x_{2}\left(\tau_{i}\right)+a x_{1}\left(\tau_{i}\right)\right), \quad t \in\left[\tau_{i}, \tau_{i+1}\right) \\
\tau_{i+1} & =\tau_{i}+r \exp \left(-\tilde{d}\left(\tau_{i}\right)\right)
\end{aligned}
$$

where $a \in \Re$ is the constant involved in (48), satisfies the UISS property with zero gain from the input $\tilde{d} \in M\left(\Re^{+}\right)$. For example, it can be verified that system (34) satisfies hypothesis (P). The vector Lyapunov function that can be used for the stability analysis is defined by the equations $V_{1}(x)=\frac{1}{2} x_{1}^{2}$ and $V_{2}(x)=\frac{1}{2}\left(x_{2}+a x_{1}\right)^{2}$, where $a \in \Re$ is the constant involved in hypothesis $(\mathrm{P})$.

Example 5.2

We consider triangular nonlinear systems of the form:

$$
\begin{aligned}
\dot{x}_{i} & =\sum_{j=1}^{i} x_{j} \phi_{i, j}\left(x_{1}, \ldots, x_{i}, d\right)+g_{i}\left(x_{1}, \ldots, x_{i}, d\right) x_{i+1}, \quad i=1, \ldots, n-1 \\
\dot{x}_{n} & =\sum_{j=1}^{n} x_{j} \phi_{n, j}\left(x_{1}, \ldots, x_{n}, d\right)+g_{n}\left(x_{1}, \ldots, x_{n}, d\right) u \\
x & =\left(x_{1}, \ldots, x_{n}\right)^{\prime} \in \Re^{n}, \quad d \in D, \quad u \in \Re
\end{aligned}
$$

where $D \subset \Re^{m}$ is a compact set, $\phi_{i, j}: \Re^{i} \times D \rightarrow \Re(j=1, \ldots, i, i=1, \ldots, n), g_{i}: \Re^{i} \times D \rightarrow \Re(i=$ $1, \ldots, n)$ locally Lipschitz mappings with $g_{i}\left(x_{1}, \ldots, x_{i}, d\right)>0$ for all $(x, d) \in \Re^{n} \times D(i=1, \ldots, n)$. A recursive method (backstepping) is presented in [39] (but also see the references therein) for the construction of a positive-definite and radially unbounded function $V \in C^{1}\left(\Re^{n} ; \Re^{+}\right)$, a mapping $\zeta \in \mathscr{N}$, a positive-definite function $W \in C^{1}\left(\Re^{n} ; \Re^{+}\right)$and a feedback function $k \in C^{0}\left(\Re^{n} ; \Re\right)$ with $k(0)=0$, such that

$$
\zeta(|e|) \leqslant V(x) \Rightarrow \nabla V(x)(F(x, d)+G(x, d) k(x+e)) \leqslant-W(x) \quad \forall x \in \Re^{n}
$$

(guarantees robustness with respect to additive measurement errors) 
or

$$
\zeta(|v|) \leqslant V(x) \Rightarrow \nabla V(x)(F(x, d)+G(x, d) k(x)+G(x, d) v) \leqslant-W(x) \quad \forall x \in \Re^{n}
$$

(guarantees robustness with respect to control actuator errors)

where

$$
\begin{aligned}
F(x, d):= & \left(x_{1} \phi_{1,1}\left(x_{1}, d\right)+g_{1}\left(x_{1}, d\right) x_{2}, \ldots, \sum_{j=1}^{n-1} x_{j} \phi_{n-1, j}\left(x_{1}, \ldots, x_{n-1}, d\right)\right. \\
& \left.+g_{n-1}\left(x_{1}, \ldots, x_{n-1}, d\right) x_{n}, \sum_{j=1}^{n} x_{j} \phi_{n, j}\left(x_{1}, \ldots, x_{n}, d\right)\right)^{\prime}
\end{aligned}
$$

$G(x, d):=\left(0, \ldots, 0, g_{n}(x, d)\right)^{\prime}$. We consider the question of whether the constructed feedback $k \in C^{0}\left(\Re^{n} ; \Re\right)$ can robustly globally asymptotically stabilize $0 \in \Re^{n}$ for system (50) when applied with zero-order hold and positive sampling rate as well as the determination of the maximum allowable sampling period for which robust global asymptotic stability is preserved.

Corollary 4.3 provides a direct answer to both issues stated above. Particularly, we have If there exists $a \in \mathscr{N}$ with $a(s)<s$ for all $s>0$ and a constant $h>0$ such that

$$
\zeta(h \rho(x)) \leqslant V(x) \text { for all } x \in \Re^{n}
$$

where

$$
\rho(x):=\max \left\{\left|F(\xi, d)+G(\xi, d) k\left(x_{0}\right)\right|: d \in D, a\left(\max \left\{V(\xi) ; V\left(x_{0}\right)\right\}\right) \leqslant V(x)\right\}
$$

in case that (51a) holds or

$$
\rho(x):=\max \left\{\left|\nabla k(\xi)\left(F(\xi, d)+G(\xi, d) k\left(x_{0}\right)\right)\right|: d \in D, a\left(\max \left\{V(\xi) ; V\left(x_{0}\right)\right\}\right) \leqslant V(x)\right\}
$$

in case that (51b) holds and $k \in C^{1}\left(\Re^{n} ; \Re\right)$, then the constructed feedback $k \in C^{0}\left(\Re^{n} ; \Re\right)$ can robustly globally asymptotically stabilize $0 \in \Re^{n}$ for system (50) when applied with zero-order hold and positive sampling rate. Moreover, the maximum allowable sampling period is the constant $h>0$ involved in (52). Specifically, the sampled-data system

$$
\begin{aligned}
\dot{x}_{i}(t)= & \sum_{j=1}^{i} x_{j}(t) \phi_{i, j}\left(x_{1}(t), \ldots, x_{i}(t), d(t)\right)+g_{i}\left(x_{1}(t), \ldots, x_{i}(t), d(t)\right) x_{i+1}(t) \\
& i=1, \ldots, n-1, \quad t \in\left[\tau_{i}, \tau_{i+1}\right) \\
\dot{x}_{n}(t)= & \sum_{j=1}^{n} x_{j}(t) \phi_{n, j}\left(x_{1}(t), \ldots, x_{n}(t), d(t)\right)+g_{n}\left(x_{1}(t), \ldots, x_{n}(t), d(t)\right) k\left(x\left(\tau_{i}\right)\right) \\
& t \in\left[\tau_{i}, \tau_{i+1}\right) \\
\tau_{i+1}= & \tau_{i}+h \exp \left(-\tilde{d}\left(\tau_{i}\right)\right)
\end{aligned}
$$

satisfies the UISS property with zero gain from the input $\tilde{d} \in M\left(\Re^{+}\right)$. The conclusion is important, since conditions (52), (53) can be easily checked after the application of the backstepping method presented in [39]. It should be emphasized that experimentation with the backstepping technique showed that it is more likely to guarantee stability for (54) when the feedback law $k \in C^{0}\left(\Re^{n} ; \Re\right)$ 
is designed to ensure robustness with respect to additive state measurement errors (i.e. following the procedure described in Chapter 6 in [39]).

\section{Example 5.3}

This example is a specialization of the results of the previous example to the case:

$$
\begin{aligned}
\phi_{i, j}\left(x_{1}, \ldots, x_{i}, d\right) & :=L d_{i, j}, \quad i=1, \ldots, n, \quad j=1, \ldots, i, \quad d_{i, j} \in[-1,1] \\
g_{i}\left(x_{1}, \ldots, x_{i}, d\right) & :=\frac{1}{2}(b-a) d_{i, i+1}+\frac{1}{2}(b+a), \quad i=1, \ldots, n, \quad d_{i,(i+1)} \in[-1,1]
\end{aligned}
$$

where $L \geqslant 0,0<a \leqslant b$ are constants and $d:=\left(d_{1,1}, d_{1,2}, d_{2,1}, d_{2,2}, d_{2,3}, \ldots, d_{n, 1}, \ldots, d_{n, n}, d_{n, n+1}\right)^{\prime} \in$ $[-1,1]^{m}, m=(((n+1)(n+2)) / 2)-1$, namely the system:

$$
\begin{aligned}
\dot{x}_{i} & =L \sum_{j=1}^{i} d_{i, j} x_{j}+\frac{1}{2}\left[(b-a) d_{i, i+1}+(b+a)\right] x_{i+1}, \quad i=1, \ldots, n-1 \\
\dot{x}_{n} & =\sum_{j=1}^{n} d_{n, j} x_{j}+\frac{1}{2}\left[(b-a) d_{n, n+1}+(b+a)\right] u \\
x & =\left(x_{1}, \ldots, x_{n}\right)^{\prime} \in \Re^{n}, \quad u \in \Re \\
d & :=\left(d_{1,1}, d_{1,2}, d_{2,1}, d_{2,2}, d_{2,3}, \ldots, d_{n, 1}, \ldots, d_{n, n}, d_{n, n+1}\right)^{\prime} \in D:=[-1,1]^{m}
\end{aligned}
$$

Using a procedure similar to the main result in [51], we may establish that there exists a symmetric, positive-definite matrix $P \in \Re^{n \times n}$, a vector $k \in \Re^{n}$ constants $\mu, c>0$ such that (51b) holds with $V(x):=x^{\prime} P x, W(x):=-\mu|x|^{2}, k(x):=k^{\prime} x$ and $\zeta(s):=c^{2} s^{2}$. Letting $a(s):=p s$ where $p \in(0,1)$, the function $\rho(x)$ defined by (53b) satisfies:

$$
\rho(x) \leqslant G \sqrt{V(x)}
$$

where $G:=|k|(n L+b \max (1,|k|)) \sqrt{n / K_{1} p}$ and $K_{1}>0$ is a constant satisfying $K_{1}|x|^{2} \leqslant x^{\prime} P x$ for all $x \in \mathfrak{R}^{n}$. Consequently, (52) will necessarily hold if the maximum allowable sampling period $h>0$ satisfies

$$
h \leqslant(c G)^{-1}
$$

For example, for $n=2$ we obtain the following parameterized family of Lyapunov functions

$$
V(x):=\frac{1}{2} x_{1}^{2}+\frac{1}{2}\left(x_{2}+r x_{1}\right)^{2} \geqslant 2+r^{2}-r \sqrt{r^{2}+4} / 2|x|^{2}
$$

where $\sigma>0, r:=(L+\sigma) / a$ and the parameterized family of vectors

$$
k:=-q\left[\frac{L+\sigma}{a}, 1\right], \quad q>\frac{(\sigma+2 b+4 L)}{4 a}+\frac{(a b+a L+L+\sigma)^{2}}{2 \sigma a^{3}}
$$

for which (51b) holds with $\zeta(s):=c^{2} s^{2}, c:=\sqrt{b / \sigma}$. It follows that the maximum allowable sampling period $h>0$ must satisfy

$$
h<\sqrt{\frac{\sigma\left(2+r^{2}-r \sqrt{r^{2}+4}\right)}{4 b q^{2}(1+r)^{2}}} \frac{1}{2 L+b \max (1, q(1+r))}
$$


For $L=1, a=1, b=2, \sigma=1, q=15$ we obtain $h<\sqrt{(3-\sqrt{8})} / 8280$. It should be emphasized again that the above estimation of the maximum allowable sampling period is conservative. However, formulae such as (59) are useful since they can assist the control practitioner to select values for the parameters which allow greater sampling periods.

The case (56) is important, since control systems of the form

$$
\begin{aligned}
& \dot{x}_{i}=f_{i}(x, u)+g_{i}(x, u) x_{i+1}, \quad i=1, \ldots, n-1 \\
& \dot{x}_{n}=f_{n}(x, u)+g_{n}(x, u) u
\end{aligned}
$$

where $f_{i}: \Re^{n} \times \Re \rightarrow \Re, g_{i}: \Re^{n} \times \Re \rightarrow \Re(i=1, \ldots, n)$ are continuous functions satisfying the following growth conditions for $i=1, \ldots, n$ :

$$
\left|f_{i}(x, u)\right| \leqslant L\left|x_{1}\right|+\cdots+L\left|x_{i}\right|, \quad a \leqslant g_{i}(x, u) \leqslant b \quad \forall(x, u) \in \Re^{n} \times \Re
$$

can be represented by the uncertain case (56). The above analysis shows that systems of the form (60) can be stabilized by means of linear sampled-data feedback with positive sampling rate. Note that no hypothesis concerning the regularity of the mappings $f_{i}: \Re^{n} \times \Re \rightarrow \Re, g_{i}: \Re^{n} \times \Re \rightarrow \Re(i=$ $1, \ldots, n)$ (e.g. the hypothesis of being globally Lipschitz) is needed.

\section{CONCLUDING REMARKS}

In the present work sufficient conditions expressed by means of single and vector Lyapunov functions of uniform input-to-output stability (UIOS) and uniform input-to-state stability (UISS) are given for finite-dimensional systems under feedback control with zero-order hold (Corollary 4.3 and Theorem 4.1, respectively). Illustrating examples are presented, which show the flexibility that a vector Lyapunov function can provide. Moreover, we show how the main results can be used in conjunction with the backstepping method for triangular nonlinear systems to check whether the feedback, constructed by the backstepping method, can robustly globally asymptotically stabilize the equilibrium point when applied with zero-order hold and positive sampling rate (emulation procedure).

It is clear that the main results of this work may be extended to the time-varying case by using time-varying Lyapunov or Razumikhin functions for time-delay systems. Moreover, the results can be extended to cover non-uniform and weighted notions of IOS and ISS (see [44, 52]). Both extensions are straightforward in the sense that the proofs follow the same procedure as the proof of Theorem 4.1.

It should be emphasized that the sufficient conditions for ISS and IOS for sampled-data systems presented in this work are not restricted to sampled-data feedback laws derived by emulation. Theorem 4.1 and Corollary 4.3 can be used for the Lyapunov redesign procedure, as explained in [12]. This topic is very important, since very rarely sampled-data feedback designs produced by emulation can guarantee global asymptotic stability. 


\section{APPENDIX A}

\section{Proof of Lemma 3.5}

Note that by virtue of Lemma 4.4 in [53], for each positive-definite continuous function $\rho: \mathfrak{R}^{+} \rightarrow \mathfrak{R}^{+}$ there exists a continuous function $\sigma$ of class $K L$, with $\sigma(s, 0)=s$ for all $s \geqslant 0$ with the following property: if $y:\left[t_{0}, t_{1}\right] \rightarrow \Re^{+}$is an absolutely continuous function and $I \subset\left[t_{0}, t_{1}\right]$ a set of Lebesgue measure zero such that $\dot{y}(t)$ is defined on $\left[t_{0}, t_{1}\right] \backslash I$ and such that the following differential inequality holds for all $t \in\left[t_{0}, t_{1}\right] \backslash I$ :

$$
\dot{y}(t) \leqslant-\rho(y(t))
$$

then the following estimate holds for all $t \in\left[t_{0}, t_{1}\right]$ :

$$
y(t) \leqslant \sigma\left(y\left(t_{0}\right), t-t_{0}\right)
$$

Actually, the statement of Lemma 4.4 in [53] does not guarantee that $\sigma$ is continuous or that $\sigma(s, 0)=s$ for all $s \geqslant 0$, but a close look at the proof of Lemma 4.4 in [53] shows that this is the case when $\rho: \Re^{+} \rightarrow \mathfrak{R}^{+}$is a positive-definite continuous function. Moreover, note that we may continuously extend $\sigma$ by defining $\sigma(s, t):=s \exp (-t)$ for $t<0$.

Clearly, (14) holds for $t=t_{0}$ and $\sigma$ the function involved in (A2). We next show that (14) holds for arbitrary $t \in\left(t_{0}, t_{1}\right]$.

Let arbitrary $t \in\left(t_{0}, t_{1}\right]$ and define the functions

$$
\tilde{u}(\tau)=\left\{\begin{array}{ll}
u(\tau) & \text { for } \tau \in\left[t_{0}, t\right] \\
0 & \text { if otherwise }
\end{array}, \quad \bar{u}(\tau):=\limsup _{\xi \rightarrow \tau} \tilde{u}(\xi)\right.
$$

Note that $\bar{u}$ is upper semi-continuous on $\tau \in\left[t_{0}, t\right]$ and consequently the function $p(\tau):=y(\tau)-\bar{u}(\tau)$ is lower semi-continuous on $\left[t_{0}, t\right]$. Next define the set

$$
A:=\left\{\tau \in\left[t_{0}, t\right]: y(\tau) \leqslant \bar{u}(\tau)\right\}
$$

We distinguish the following cases:

(1) $A=\emptyset$ : In this case we have $y(\tau)>\bar{u}(\tau)$ for all $\tau \in\left[t_{0}, t\right]$. Since $\bar{u}(\tau) \geqslant u(\tau)$ for all $\tau \in\left[t_{0}, t\right]$, the previous inequality in conjunction with (13) implies that $\dot{y}(\tau) \leqslant-\rho(y(\tau))$ for all $\tau \in\left[t_{0}, t\right] \backslash I$. Thus, in this case Lemma 4.4 in [53] guarantees that estimate (A2) holds.

(2) $A \neq \emptyset$ and $\xi:=\sup A<t$ : In this case there exists a sequence $\tau_{i} \leqslant \xi$ with $\tau_{i} \rightarrow \xi$ and $y\left(\tau_{i}\right)-$ $\bar{u}\left(\tau_{i}\right) \leqslant 0$. Since the function $p(t)=y(t)-\bar{u}(t)$ is lower semi-continuous, we obtain $p(\xi)=$ $\liminf _{\tau \rightarrow \xi} p(\tau) \leqslant 0$ and consequently $y(\xi) \leqslant \bar{u}(\xi)$. Moreover, note that by virtue of definition (A3), (13) and since $\bar{u}(\tau) \geqslant u(\tau)$ for all $\tau \in\left[t_{0}, t\right]$, the differential inequality $\dot{y}(\tau) \leqslant-\rho(y(\tau))$ holds for all $\tau \in(\xi, t] \backslash I$. Consequently, Lemma 4.4 in [53] implies $y(t) \leqslant \sigma(y(\tau), t-\tau)$ for all $\tau \in(\xi, t]$. By virtue of continuity of $\sigma$ and $y$ we obtain

$$
y(t) \leqslant \sigma(y(\xi), t-\xi)
$$

which combined $y(\xi) \leqslant \bar{u}(\xi)$ directly implies

$$
y(t) \leqslant \sigma(\bar{u}(\xi), t-\xi) \leqslant \sup _{t_{0} \leqslant s \leqslant t} \sigma(\bar{u}(s), t-s)
$$


(3) $A \neq \emptyset$ and $\xi:=\sup A=t$. In this case there exists a sequence $\tau_{i} \leqslant t$ with $\tau_{i} \rightarrow t$ and $y\left(\tau_{i}\right)-$ $\bar{u}\left(\tau_{i}\right) \leqslant 0$. Since the function $p(t)=y(t)-\bar{u}(t)$ is lower semi-continuous, we obtain $p(t)=$ $\liminf _{\tau \rightarrow t} p(\tau) \leqslant 0$ and consequently $y(t) \leqslant \bar{u}(t)$. Moreover, since $\sigma(s, 0)=s$ for all $s \geqslant 0$, it holds that $y(t) \leqslant u(t)=\sigma(\bar{u}(t), 0) \leqslant \sup _{t_{0} \leqslant s \leqslant t} \sigma(\bar{u}(s), t-s)$.

Combining all the above cases, we may conclude that

$$
y(t) \leqslant \max \left\{\sigma\left(y\left(t_{0}\right), t-t_{0}\right), \sup _{t_{0} \leqslant s \leqslant t} \sigma(\bar{u}(s), t-s)\right\}
$$

Let $M:=\sup _{t_{0} \leqslant s \leqslant t} u(s)$. For each $\varepsilon>0$, there exists $\delta>0$ such that $\sigma(s, \tau-\delta)-\sigma(s, \tau)<\varepsilon$ for all $(s, \tau) \in[0, M] \times[0, t]$. Note that since

$$
\bar{u}(\tau):=\limsup _{\xi \rightarrow \tau} \tilde{u}(\xi) \quad \text { and } \quad \tilde{u}(\tau)= \begin{cases}u(\tau) & \text { for } \tau \in\left[t_{0}, t\right] \\ 0 & \text { if otherwise }\end{cases}
$$

it follows that $\bar{u}(s) \leqslant \sup \left\{u(r): \max \left(s-\delta, t_{0}\right) \leqslant r \leqslant \min (s+\delta, t)\right\}$, for all $s \in\left[t_{0}, t\right]$. The previous inequalities imply

$$
\begin{aligned}
\sigma(\bar{u}(s), t-s) & \leqslant \sup \left\{\sigma(u(r), t-s): \max \left(s-\delta, t_{0}\right) \leqslant r \leqslant \min (s+\delta, t)\right\} \\
& \leqslant \sup \left\{\sigma(u(r), t-r-\delta): \max \left(s-\delta, t_{0}\right) \leqslant r \leqslant \min (s+\delta, t)\right\} \\
& \leqslant \sup \left\{\sigma(u(r), t-r): \max \left(s-\delta, t_{0}\right) \leqslant r \leqslant \min (s+\delta, t)\right\}+\varepsilon \\
& \leqslant \sup _{t_{0} \leqslant r \leqslant t} \sigma(u(r), t-r)+\varepsilon
\end{aligned}
$$

The above inequality in conjunction with (A5) imply that for each $\varepsilon>0$, it holds that

$$
y(t) \leqslant \max \left\{\sigma\left(y\left(t_{0}\right), t-t_{0}\right), \sup _{t_{0} \leqslant s \leqslant t} \sigma(u(s), t-s)\right\}+\varepsilon
$$

Since $\varepsilon>0$ is arbitrary, we conclude that the above estimate directly implies (14). The proof is complete.

\section{REFERENCES}

1. Monaco S, Normand-Cyrot D. Issues on nonlinear digital control. European Journal of Control 2001; 7:160-177.

2. Grune L, Nesic D. Optimization based stabilization of sampled-data nonlinear systems via their approximate discrete-time models. SIAM Journal on Control and Optimization 2003; 42:98-122.

3. Kazantzis N, Kravaris C. System-theoretic properties of sampled-data representations of nonlinear systems obtained via Taylor-Lie series. International Journal of Control 1997; 67(6):997-1020.

4. Laila DS, Nesic D, Teel AR. Open and closed-loop dissipation inequalities under sampling and controller emulation. European Journal of Control 2002; 18:109-125.

5. Laila DS, Astolfi A. Input-to-state stability for discrete-time time-varying systems with applications to robust stabilization of systems in power form. Automatica 2005; 41:1891-1903.

6. Nesic D, Teel AR, Sontag ED. Formulas relating KL stability estimates of discrete-time and sampled-data nonlinear systems. Systems and Control Letters 1999; 38(1):49-60.

7. Nesic D, Teel AR, Kokotovic PV. Sufficient conditions for stabilization of sampled-data nonlinear systems via discrete-time approximations. Systems and Control Letters 1999; 38(4-5):259-270. 
8. Nesic D, Teel AR. Sampled-data control of nonlinear systems: an overview of recent results. In Perspectives on Robust Control, Moheimani RSO (ed.). Springer: New York, 2001; 221-239.

9. Nesic D, Laila DS. A note on input-to-state stabilization for nonlinear sampled-data systems. IEEE Transactions on Automatic Control 2002; 47:1153-1158.

10. Nesic D, Angeli D. Integral versions of ISS for sampled-data nonlinear systems via their approximate discrete-time models. IEEE Transactions on Automatic Control 2002; 47:2033-2038.

11. Nesic D, Teel A. A framework for stabilization of nonlinear sampled-data systems based on their approximate discrete-time models. IEEE Transactions on Automatic Control 2004; 49(7):1103-1122.

12. Nesic D, Grune L. Lyapunov-based continuous-time nonlinear controller redesign for sampled-data implementation. Automatica 2005; 41:1143-1156.

13. Zaccarian L, Teel AR, Nesic D. On finite gain $\mathscr{L}_{p}$ stability of nonlinear sample-data systems. Systems and Control Letters 2003; 49:201-212.

14. Kellett CM, Shim H, Teel AR. Further results on robustness of (possibly discontinuous) sample and hold feedback. IEEE Transactions on Automatic Control 2004; 49(7):1081-1089.

15. Khalil HK. Performance recovery under output feedback sampled-data stabilization of a class of nonlinear systems. IEEE Transactions on Automatic Control 2004; 49(12):2173-2184.

16. Grune L. Homogeneous state feedback stabilization of homogenous systems. SIAM Journal on Control and Optimization 2000; 38(4):1288-1308.

17. Herrmann G, Spurgeon SK, Edwards C. Discretization of sliding mode based control schemes. Proceedings of the 38th Conference on Decision and Control, Phoenix, AZ, U.S.A., 1999; 4257-4262.

18. Bernstein DS, Hollot CV. Robust stability for sampled-data control systems. Systems and Control Letters 1989; 13:217-226.

19. Sontag ED. Mathematical Control Theory (2nd edn). Springer: New York, 1998.

20. Hu B, Michel AN. Stability analysis of digital control systems with time-varying sampling periods. Automatica 2000; 36:897-905.

21. $\mathrm{Hu} \mathrm{B}$, Michel AN. Robustness analysis of digital control systems with time-varying sampling periods. Journal of the Franklin Institute 2000; 337:117-130.

22. Mancilla-Aguilar JL, Garcia RA, Troparevsky MI. Stability of a certain class of hybrid dynamical systems. International Journal of Control 2000; 73(15):1362-1374.

23. Ye H, Michel AN, Hou L. Stability theory for hybrid dynamical systems. IEEE Transactions on Automatic Control 1998; 43(4):461-474.

24. Artstein Z, Weiss G. State nullification by memoryless output feedback. Mathematics of Control, Signals and Systems 2005; 17:38-56.

25. Monaco S, Normand-Cyrot D. Zero dynamics of sampled nonlinear systems. Systems and Control Letters 1988; 11:229-234.

26. Monaco S, Normand-Cyrot D. A unified representation for nonlinear discrete-time and sampled dynamics. Journal of Mathematical Systems, Estimation, and Control 1997; 7(4):477-503.

27. Nesic D, Teel AR, Carnevale D. Explicit computation of the sampling period in emulation of controllers for nonlinear sampled-data systems. IEEE Transactions on Automatic Control, in press.

28. Fridman E, Seuret A, Richard J-P. Robust sampled-data stabilization of linear systems: an input-delay approach. Automatica 2004; 40:1441-1446.

29. Fridman E, Shaked U, Suplin V. Input/output delay approach to robust sampled-data $H_{\infty}$ control. Systems and Control Letters 2005; 54:271-282.

30. Hale JK, Lunel SMV. Introduction to Functional Differential Equations. Springer: New York, 1993.

31. Niculescu SI. Delay Effects on Stability, A Robust Control Approach. Springer: Heidelberg, Germany, 2001.

32. Teel AR. Connections between Razumikhin-type theorems and the ISS nonlinear small gain theorem. IEEE Transactions on Automatic Control 1998; 43(7):960-964.

33. Nersesov SG, Haddad WM. On the stability and control of nonlinear dynamical systems via vector Lyapunov functions. IEEE Transactions on Automatic Control 2006; 51(2):203-215.

34. Sontag ED. Smooth stabilization implies coprime factorization. IEEE Transactions on Automatic Control 1989; 34:435-443.

35. Sontag ED, Wang Y. On characterizations of the input-to-state stability property. Systems and Control Letters 1995; 24:351-359.

36. Sontag ED, Wang Y. New characterizations of the input-to-state stability. IEEE Transactions on Automatic Control 1996; 41:1283-1294. 
37. Sontag ED, Wang Y. Notions of input to output stability. Systems and Control Letters 1999; 38:235-248.

38. Sontag ED, Wang Y. Lyapunov characterizations of input-to-output stability. SIAM Journal on Control and Optimization 2001; 39:226-249.

39. Freeman RA, Kokotovic PV. Robust Nonlinear Control Design-State Space and Lyapunov Techniques. Birkhauser: Boston, 1996.

40. Clarke FH, Ledyaev YS, Sontag ED, Subbotin AI. Asymptotic controllability implies feedback stabilization. IEEE Transactions on Automatic Control 1997; 42(10):1394-1407.

41. Sontag ED. Clocks and insensitivity to small measurement errors. ESAIM: Control, Optimisation and Calculus of Variations 1999; 4:537-557.

42. Grune L. Stabilization by sampled and discrete feedback with positive sampling rate. In Stability and Stabilization of Nonlinear Systems, Aeyels D, Lamnabhi-Lagarrigue F, van der Schaft A (eds). Springer: London, 1999; 165-182.

43. Tabuada P. Event-triggered real-time scheduling of stabilizing control tasks. IEEE Transactions on Automatic Control 2007; 52(9):1680-1685.

44. Karafyllis I. A system-theoretic framework for a wide class of systems, I: applications to numerical analysis. Journal of Mathematical Analysis and Applications 2007; 328(2):876-899.

45. Khalil HK. Nonlinear Systems (2nd edn). Prentice-Hall: Englewood Cliffs, NJ, 1996.

46. Stuart AM, Humphries AR. Dynamical Systems and Numerical Analysis. Cambridge University Press: Cambridge, 1998.

47. Karafyllis I. The non-uniform in time small-gain theorem for a wide class of control systems with output. European Journal of Control 2004; 10(4):307-323.

48. Sontag ED, Ingalls B. A small-gain theorem with applications to input/output systems, incremental stability, detectability, and interconnections. Journal of the Franklin Institute 2002; 339:211-229.

49. Jiang ZP, Teel A, Praly L. Small-gain theorem for ISS systems and applications. Mathematics of Control, Signals and Systems 1994; 7:95-120.

50. Grune L. Input-to-state dynamical stability and its Lyapunov function characterization. IEEE Transactions on Automatic Control 2002; 47:1499-1504.

51. Tsinias J. A theorem on global stabilization of nonlinear systems by linear feedback. Systems and Control Letters $1991 ; \mathbf{1 7}: 357-362$.

52. Karafyllis I. A system-theoretic framework for a wide class of systems, II: input-to-output stability. Journal of Mathematical Analysis and Applications 2007; 328(1):466-486.

53. Lin Y, Sontag ED, Wang Y. A smooth converse Lyapunov theorem for robust stability. SIAM Journal on Control and Optimization 1996; 34:124-160. 